Preprint typeset in JHEP style - HYPER VERSION

\title{
New generalized nonspherical black hole solutions
}

\author{
Burkhard Kleihaus ${ }^{\dagger}$, Jutta Kunz ${ }^{\dagger}$, Eugen Radu^» and Maria J. Rodriguez \\ ${ }^{\dagger}$ Institut für Physik, Universität Oldenburg, Postfach 2503 D-26111 Oldenburg, Germany \\ $¥$ Max-Planck-Institut für Gravitationsphysik, Albert-Einstein-Institut, 14476 Golm, \\ Germany \\ ${ }^{\star}$ Department of Computer Science, National University of Ireland Maynooth, Maynooth, \\ Ireland \\ ${ }^{\diamond}$ School of Theoretical Physics - DIAS, 10 Burlington Road, Dublin 4, Ireland
}

\begin{abstract}
We present numerical evidence for the existence of several types of static black hole solutions with a nonspherical event horizon topology in $d \geq 6$ spacetime dimensions. These asymptotically flat configurations are found for a specific metric ansatz and can be viewed as higher dimensional counterparts of the $d=5$ static black rings, dirings and black Saturn. Similar to that case, they are supported against collapse by conical singularities. The issue of rotating generalizations of these solutions is also considered.
\end{abstract}

KEYWORDS: black holes, numerical solutions. 


\section{Contents}

1. Introduction 2

2. The general formalism 3

2.1 The field equations and a metric ansatz 3

2.2 Known solutions

2.2.1 Minkowski space-time 5

2.2.2 Schwarschild-Tangherlini black hole 5

2.3 The rod structure of black hole solutions 6

2.3.1 Five dimensional structure 6

2.3.2 Higher dimensional structure 8

2.3.3 Physical quantities 10

2.4 Numerical procedure 14

2.5 The issue of $d>5$ black ring solutions 16

3. General results for static black holes

3.1 Uni-horizon black holes 17

3.1.1 Schwarzschild-Tangherlini black hole: $S^{d-2}$ horizon 17

3.1 .2 Generalized black ring: $S^{2} \times S^{d-4}$ horizon 18

3.2 Multi-horizon black holes 20

3.2.1 Generalized black Saturn: $\left(S^{2} \times S^{d-4}\right) \times S^{d-2}$ horizon 20

3.2 .2 Generalized di-rings: $\left(S^{2} \times S^{d-4}\right) \times\left(S^{2} \times S^{d-4}\right)$ horizon

4. Remarks on rotating black holes with a nonspherical horizon topology 26

4.1. A generalized metric ansatz 26

4.1.1 The singly spinning Myers-Perry black hole 27

4.1.2 A rotating $S^{2}: d=6$ black holes with $S^{2} \times S^{2}$ topology of the horizon 28

4.2 A rotating $S^{d-4}$

4.2.1 A general metric ansatz 29

$4.2 .2 d=7$ rotating solutions with $S^{2} \times S^{3}$ horizon topology 30

5. Conclusions

A. Five-dimensional seeds 34

A.1 The static black ring 34

A.2 The static Saturn 35

A.3 The static di-ring 36 
B. 'Isotropic' coordinates and new diagrams

B.1 A coordinate transformation 38

B.2 $d=5$ static solutions in 'isotropic' coordinates 39

B.3 Rotating solutions: balanced black ring and Myers-Perry black hole 42

\section{Introduction}

In recent years the interest in the properties of gravity in more than $d=4$ dimensions has increased significantly. This interest was enhanced by the development of string theory, which requires a ten-dimensional spacetime, to be consistent from a quantum point of view. An unexpected result in this area was Emparan and Reall's discovery of the black ring in $d=5$ spacetime dimensions [1], 2]. This asymptotically flat solution of the Einstein equations has a horizon with topology $S^{2} \times S^{1}$, while the Myers-Perry black hole [3] has a horizon topology $S^{3}$. This made clear that a number of well known results in $d=4$ gravity do not have a simple extension to higher dimensions. For example, the $d=5$ gravity allows for multi-black hole configurations regular outside and on the horizon. In this case, at least one of the constituents possesses a nonspherical topology of the horizon, the simplest examples being the black Saturn 㭵 (a black ring with a central black hole), a diring [5, 6] (two concentric coplanar black rings) and bicyling black rings [7] (two black rings in orthogonal planes).

However, while one can construct an encyclopedia of general relativity exact solutions in four and five dimensions, the situation for $d>5$ is more patchy (see e.g. [8]). For most of the cases, the known solutions are very special, with a large amount of symmetry. Moreover, it becomes clear that as the dimension increases, the phase structure of the solutions becomes increasingly intricate and diverse. The main obstacle stopping the progress in this field seems to be the absence of closed form solutions (apart from the Myers-Perry black holes), which were very useful in $d=5$. No general framework seems to exist for $d>5$, and the issue of constructing black objects with a nonspherical horizon topology was considered by using various approximations or numerical methods. Most of the results in this area have been found by using the method of matched asymptotic expansions [9, 10]. The central assumption is that some black objects, in certain ultra-spinning regimes, can be approximated by very thin black strings or branes curved into a given shape. However, this method has limitations; black holes whith no black membrane behavior (e.g. at high spins) would not be captured by this approach [11.

Although it would clearly be preferable to have analytic solutions ${ }^{1}$, some of the $d>5$ black holes with a nonspherical horizon topology can be constructed numerically, within a nonperturbative approach, as solutions of partial differential equations with suitable boundary conditions.

\footnotetext{
${ }^{1}$ However, one should not exclude the possibility that most of these solutions will remain analytically intractable within a nonperturbative approach.
} 
The main purpose of this paper is to present a general framework for a special class of static configurations with a symmetry group $R_{t} \times U(1) \times S O(d-3)$ and to present numerical evidence for the existence of such solutions with nonspherical horizon topology. For $d=5$, this framework reduces to that used in [2] to construct generalized Weyl solutions. However, for higher values of the spacetime dimension, the solutions can be found only numerically. We argue that the basic properties of the $d=5$ case still hold for $d>5$ configurations with a symmetry group $R_{t} \times U(1) \times S O(d-3)$, in particular the rod structure of the solutions. The simplest example of a $d>5$ black object with a nonspherical horizon obtained within this approach was studied in Ref. [12 and has a horizon topology $S^{2} \times S^{d-4}$. In this work, on the one hand, we extend these results and discuss the basic features of two new types of configurations representing composite black objects with $\left(S^{2} \times S^{d-4}\right) \times S^{d-2}-$ a generalized black Saturn - and also $\left(S^{2} \times S^{d-4}\right) \times\left(S^{2} \times S^{d-4}\right)$ horizons -a generalized diring. On the other hand, within a slightly more general metric ansatz, we consider rotating solutions in either the $S^{2}$ or the $S^{d-4}$ spheres.

This paper is organized as follows: in the next Section we present a systematic discussion of this approach together with its limitations, while in Section III we present our numerical results. All solutions are found within a nonperturbative approach, by directly solving Einstein equations which for our ansatz reduce to a set of four nonlinear partial differential equations.

Since all these solutions are plagued by conical singularities which seem to be unavoidable in the absence of rotation, the issue of spinning solutions is addressed in Section IV. The results reported there are only partial, and so far we could not construct spinning balanced solutions. However, we expect that they will be useful for further work in this direction.

Also, we have found that all new static solutions in this work have similar qualitative properties as their five dimensional counterparts. Therefore in the Appendix A we present the basic properties of the corresponding $d=5$ solutions, which are known in closed form. Appendix B introduces a new coordinate system which simplifies the numerical calculations and leads to high accuracy (e.g. it has allowed to recover numerically the spinning balanced black ring starting with the static solution).

\section{The general formalism}

\subsection{The field equations and a metric ansatz}

We consider the Einstein action

$$
I=\frac{1}{16 \pi G_{d}} \int_{\mathcal{M}} d^{d} x \sqrt{-g} R-\frac{1}{8 \pi G_{d}} \int_{\partial \mathcal{M}} d^{d-1} x \sqrt{-h} K,
$$

in a $d$-dimensional spacetime, with $d \geq 5$. The last term in (2.1) is the Hawking-Gibbons surface term [13], which is required in order to have a well-defined variational principle. $K$ is the trace of the extrinsic curvature for the boundary $\partial \mathcal{M}$ and $h$ is the induced metric of the boundary. Also, $G_{d}$ is Newton's constant in $d$-dimensions; for simplicity, we shall set $G_{d}=1$ in this work. 
The upshot for our computations is that the line element of the static solutions of interest can be cast in the following form (where $0 \leq \psi \leq 2 \pi, 0 \leq \theta \leq \pi / 2$ and $d \Omega_{d-4}^{2}$ the unit metric on $S^{d-4}$, while $\left.0 \leq \rho<\infty,-\infty<z<\infty\right)$

$$
d s^{2}=-e^{2 U_{0}(\rho, z)} d t^{2}+e^{2 \nu(\rho, z)}\left(d \rho^{2}+d z^{2}\right)+e^{2 U_{1}(\rho, z)} d \psi^{2}+e^{2 U_{2}(\rho, z)} d \Omega_{d-4}^{2} .
$$

The solutions constructed within this ansatz are static and axisymmetric, with a symmetry group $R_{t} \times U(1) \times S O(d-3)$ (where $R_{t}$ denotes the time translation). While in principle it is possible to choose any kind of boundary conditions, we will only concentrate on black hole solutions which asymptote to flat spacetime. Moreover, the coordinates in (2.2) have a rectangular boundary and thus are suitable for numerical calculations.

A suitable combination of the Einstein equations, $G_{t}^{t}=0, G_{\rho}^{\rho}+G_{z}^{z}=0, G_{\psi}^{\psi}=0$ and $G_{\varphi}^{\varphi}=0$ (with $\varphi$ an angle on $\Omega_{d-4}$ ), yields the following set of equations for the functions $U_{0}, U_{1}, U_{2}$

$$
\begin{aligned}
& \nabla^{2} U_{0}+\left(\nabla U_{0}\right)^{2}+\left(\nabla U_{0}\right) \cdot\left(\nabla U_{1}\right)+(d-4)\left(\nabla U_{0}\right) \cdot\left(\nabla U_{2}\right)=0, \\
& \nabla^{2} U_{1}+\left(\nabla U_{1}\right)^{2}+\left(\nabla U_{0}\right) \cdot\left(\nabla U_{1}\right)+(d-4)\left(\nabla U_{1}\right) \cdot\left(\nabla U_{2}\right)=0, \\
& \nabla^{2} U_{2}+(d-4)\left(\nabla U_{2}\right)^{2}+\left(\nabla U_{0}\right) \cdot\left(\nabla U_{2}\right)+\left(\nabla U_{1}\right) \cdot\left(\nabla U_{2}\right)-(d-5) e^{2 \nu-2 U_{2}}=0,
\end{aligned}
$$

and

$$
\begin{aligned}
\nabla^{2} \nu-\left(\nabla U_{0}\right) \cdot\left(\nabla U_{1}\right)-(d-4)\left(\nabla U_{0}\right) & \cdot\left(\nabla U_{2}\right)-(d-4)\left(\nabla U_{1}\right) \cdot\left(\nabla U_{1}\right) \\
+ & \frac{1}{2}(d-4)(d-5)\left(e^{2 \nu-2 U_{2}}-\left(\nabla U_{2}\right)^{2}\right)=0,
\end{aligned}
$$

for the metric function $\nu$, where we define

$$
(\nabla U) \cdot(\nabla V)=\partial_{\rho} U \partial_{\rho} V+\partial_{z} U \partial_{z} V, \quad \nabla^{2} U=\partial_{\rho}^{2} U+\partial_{z}^{2} U
$$

The remaining Einstein equations $G_{z}^{\rho}=0, G_{\rho}^{\rho}-G_{z}^{z}=0$ yield two constraints. Following [14], we note that setting $G_{t}^{t}=G_{\varphi}^{\varphi}=G_{\rho}^{\rho}+G_{z}^{z}=0$ in $\nabla_{\mu} G^{\mu \rho}=0$ and $\nabla_{\mu} G^{\mu z}=0$, we obtain the Cauchy-Riemann relations

$$
2 \partial_{z}\left(\sqrt{-g} G_{z}^{\rho}\right)+\partial_{\rho}\left(\sqrt{-g}\left(G_{\rho}^{\rho}-G_{z}^{z}\right)\right)=0,2 \partial_{\rho}\left(\sqrt{-g} G_{z}^{\rho}\right)-\partial_{z}\left(\sqrt{-g}\left(G_{\rho}^{\rho}-G_{z}^{z}\right)\right)=0 .(2
$$

Thus, the weighted constraints satisfy Laplace equations, and the constraints are fulfilled when one of them is satisfied on the boundary and the other at a single point [14]. As we shall see, this is the case for all configurations discussed in the next Section.

Although the Einstein equations take a simple form in terms of $\left(U_{i}, \nu\right)$, for the purposes of this paper it is more convenient to work with a set a functions $f_{i}$ defined as follows ${ }^{2}$

$$
e^{2 \nu(\rho, z)}=f_{1}(\rho, z), \quad e^{2 U_{2}(\rho, z)}=f_{2}(\rho, z), \quad e^{2 U_{3}(\rho, z)}=f_{3}(\rho, z), \quad e^{2 U_{1}(\rho, z)}=f_{0}(\rho, z)
$$

This leads to a line element

$$
d s^{2}=-f_{0}(\rho, z) d t^{2}+f_{1}(r, z)\left(d \rho^{2}+d z^{2}\right)+f_{2}(\rho, z) d \psi^{2}+f_{3}(\rho, z) d \Omega_{d-4}^{2},
$$

\footnotetext{
${ }^{2}$ Some divergencies are avoided in this way. For example, $f_{i} \rightarrow 0$ would correspond to $U_{i} \rightarrow-\infty$ which is clearly not suitable for a numerical approach.
} 
which was used in our numerical study of the $d>5$ solutions.

One might be concerned that (2.8) is too restrictive to leave room for new interesting black hole solutions. In higher dimensions, a priori this is not the $\operatorname{case}^{3}$, and, in the next Section, we shall present numerical evidence for the existence of nontrivial solutions which share the basic properties of some $d=5$ configurations with a nonspherical horizon topology.

Other more general metric proposals which may describe higher dimensional black hole solutions with a nonspherical horizon topology have been presented in [17]. However, due to their complexity, they will be out of our present scope.

\subsection{Known solutions}

\subsubsection{Minkowski space-time}

In $d \geq 5$ dimensions, the flat spacetime metric can be written in the form (where $0 \leq r<\infty$, $0 \leq \psi \leq 2 \pi, 0 \leq \theta \leq \pi / 2)$

$$
d s^{2}=-d t^{2}+d r^{2}+r^{2}\left(d \theta^{2}+\sin ^{2} \theta d \psi^{2}+\cos ^{2} \theta d \Omega_{d-4}^{2}\right)
$$

thus with the metric on $S^{d-2}$ written in terms of a warped product of $S^{2}$ and $S^{d-4}$. Then, for all dimensions, the coordinate transformation

$$
r=\sqrt{2}\left(\rho^{2}+z^{2}\right)^{1 / 4}, \quad \theta=\frac{1}{2} \arctan (\rho / z)
$$

leads to the equivalent form of (2.9)

$d s^{2}=-d t^{2}+\frac{1}{2 \sqrt{\rho^{2}+z^{2}}}\left(d \rho^{2}+d z^{2}\right)+\left(\sqrt{\rho^{2}+z^{2}}+z\right) d \psi^{2}+\left(\sqrt{\rho^{2}+z^{2}}-z\right) d \Omega_{d-4}^{2}$,

where $0 \leq \rho<\infty,-\infty<z<\infty$.

An interesting observation here concerns the value of the determinant $\Delta$ for the nonconformal part of the metric ${ }^{4}$ (i.e. the line element (2.8) without the $(\rho, z)$-part). One can see that even for the simplest case of a Minkowski space-time within the parametrization (2.8), $\Delta=-\rho^{2}$ for $d=4,5$ only.

\subsubsection{Schwarschild-Tangherlini black hole}

The simplest example of a $d>5$ nontrivial solution that can be studied within this approach corresponds to the Schwarzschild-Tangherlini black hole. Usually this metric is written in the form

$$
d s^{2}=-f(r) d t^{2}+f(r)^{-1} d r^{2}+r^{2}\left(d \theta^{2}+\sin ^{2} \theta d \psi^{2}+\cos ^{2} \theta d \Omega_{d-4}^{2}\right),
$$

\footnotetext{
${ }^{3}$ Black holes have to be of positive Yamabe type 15] and, if stationary, they have to be axisymmetric 16.

${ }^{4}$ The choice of this determinant has been proven to be crucial in recent progress on finding new classes of solutions [2] and also for the metric proposals in [17].
} 
with $f(r)=1-\mu / r^{d-3}$. This $d$-dimensional static black hole solution has an isometry group $R_{t} \times S O(d-1)$. By a change of coordinates one can bring the metric to the desired conformal form (2.8). The change of coordinates is

$$
\rho=\frac{\alpha}{2} \sin 2 \theta \sinh G(r), \quad z=\frac{\alpha}{2} \cos 2 \theta \cosh G(r),
$$

which yields

$$
d \rho^{2}+d z^{2}=\frac{\alpha^{2}}{2}(\cosh 2 G(r)-\cos 4 \theta)\left(\frac{G^{\prime}(r)^{2}}{4} d r^{2}+d \theta^{2}\right) .
$$

By simply integrating $G(r)$ one finds

$$
G(r)=2 \int \sqrt{\frac{g_{r r}}{g_{\theta \theta}}} d r=2 \int \frac{1}{\sqrt{f(r) r^{2}}} d r=\log \left[2^{\frac{4}{d-3}}\left(r^{\frac{d-3}{2}}+\sqrt{r^{d-3}-\mu}\right)^{\frac{4}{d-3}}\right]+k,
$$

where $k=-\frac{2}{d-3} \log (4 \mu)$ is the integration constant. And, finally we fix $\alpha=2^{\frac{d-7}{d-3}} \mu^{\frac{2}{d-3}}$ to match asymptotically flat space.

The transformation (2.13) simplifies drastically in $d=5$

$$
\rho=\frac{1}{2} \sin 2 \theta\left(1-\frac{\mu}{r^{2}}\right)^{1 / 2} r^{2}, \quad z=\frac{1}{2} \cos 2 \theta\left(1-\frac{\mu}{2 r^{2}}\right) r^{2},
$$

matching the findings of [2], and in $d=7$ where

$$
\rho=\frac{1}{2} \sin 2 \theta\left(1-\frac{\mu}{r^{4}}\right)^{1 / 2} r^{2}, \quad z=\frac{1}{2} \cos 2 \theta r^{2} .
$$

A straightforward but cumbersome computation leads to the following expression for the Schwarzschild-Tangherlini black hole in the $(\rho, z)$ coordinates

$$
\begin{aligned}
d s^{2}= & -\left(\frac{v^{(d-3) / 2}-1}{v^{(d-3) / 2}+1}\right)^{2} d t^{2}+\frac{\mu\left(v^{(d-3) / 2}+1\right)^{4 /(d-3)}}{4 v\left(z^{2} \frac{\left(v^{2}-1\right)^{2}}{\left(v^{2}+1\right)^{2}}+\rho^{2} \frac{\left(v^{2}+1\right)^{2}}{\left(v^{2}-1\right)^{2}}\right)}\left(d \rho^{2}+d z^{2}\right) \\
& +\frac{\left(v^{(d-3) / 2}+1\right)^{\frac{4}{d-3}}}{2 v\left(v^{2}+1\right)}\left(\mu\left(v^{2}+1\right)+2 z v\right) d \psi^{2}+\frac{\left(v^{(d-3) / 2}+1\right)^{\frac{4}{d-3}}}{2 v\left(v^{2}+1\right)}\left(\mu\left(v^{2}+1\right)-2 z v\right) d \Omega_{d-4}^{2},
\end{aligned}
$$

where

$$
\begin{aligned}
& v=\frac{1}{\mu}\left(\rho^{2}+z^{2}+\mathcal{P}+\sqrt{2} \sqrt{\left(\rho^{2}+z^{2}\right)^{2}+\mu^{2}\left(\rho^{2}-z^{2}\right)+\left(\rho^{2}+z^{2}\right) \mathcal{P}}\right)^{1 / 2}, \\
& \text { and } \mathcal{P}=\sqrt{\mu^{4}+2 \mu^{2}\left(\rho^{2}-z^{2}\right)+\left(\rho^{2}+z^{2}\right)^{2}} .
\end{aligned}
$$

Moreover, one can also show that for $d=5$ these expressions reduce to those in [2].

\subsection{The rod structure of black hole solutions}

\subsubsection{Five dimensional structure}

For $d=5$, the coordinates in (2.9) are the usual Weyl coordinates, while the sphere $\Omega_{d-4}$ reduces to a single angular coordinate $\varphi$, with $0 \leq \varphi \leq 2 \pi$. 
In this case, it is most convenient to choose the three functions $U_{i}$ as to satisfy the condition

$$
\sum_{i} U_{i}=\log \rho
$$

This is compatible with the vacuum Einstein equations (2.3), which for the choice (2.19) imply also

$$
\frac{\partial^{2} U_{i}}{\partial \rho^{2}}+\frac{1}{\rho} \frac{\partial U_{i}}{\partial \rho}+\frac{\partial^{2} U_{i}}{\partial z^{2}}=0
$$

One can see that $(\sqrt{2.20})$ is just Laplace's equation in a (fictious) three-dimensional flat space with metric $d s^{2}=d \rho^{2}+\rho^{2} d \Theta^{2}+d z^{2}$, whose solutions are well-known.

From the other components of the Einstein equations $G_{\rho}^{\rho}-G_{z}^{z}=0$ and $G_{\rho}^{z}=0$, we obtain the equations which determine the function $\nu(\rho, z)$ for a given solution of the equation (2.20)

$$
\nu^{\prime}=-\frac{1}{2 \rho}+\frac{\rho}{2}\left(U_{1}^{\prime 2}+U_{2}^{\prime 2}+U_{3}^{\prime 2}-\dot{U}_{1}^{2}-\dot{U}_{2}^{\prime 2}-\dot{U}_{3}^{2}\right), \quad \dot{\nu}=\rho\left(\dot{U}_{1}^{\prime}+\dot{U}_{2}^{\prime}+\dot{U}_{3}^{\prime}\right),
$$

where a prime denotes the derivative with respect to $\rho$ and a dot denotes the derivative with respect to $z$. Solutions with the ansatz (2.2) and with $U_{1}, U_{2}, U_{3}$ and $\nu$ satisfying the equations (2.20), (2.21) are usually called generalized Weyl solutions [2].

This approach has proven very fruitful, a variety of physically interesting configurations being discussed in the literature. They can be uniquely characterized by the boundary conditions on the $z$-axis, known as the rod-structure [2, 18, 19]. One finds that the physically relevant solutions for $U_{i}$ can also be thought of as Newtonian potentials produced by thin rods of zero thickness with linear mass density $1 / 2$, placed on the axis of symmetry in the auxiliary three-dimensional flat space. Then the constraint (2.19) states that these sources must add up to give an infinite rod.

In this approach, the $z$-axis is divided into $N$ intervals (called rods of the solution), $\left[-\infty, z_{1}\right],\left[z_{1}, z_{2}\right], \ldots,\left[z_{N-1}, \infty\right]$. As proven in [18], in order to avoid curvature naked singularities at $\rho=0$, it is a necessary condition that only one of the functions $f_{0}(0, z), f_{2}(0, z)$, $f_{3}(0, z)$ becomes zero for a given rod, except for isolated points between the intervals.

For the static case discussed here, a horizon corresponds to a timelike rod where $f_{0}(0, z)=0$ while $\lim _{\rho \rightarrow 0} f_{0}(\rho, z) / \rho^{2}>0$. There are also spacelike rods corresponding to compact directions specified by the conditions $f_{a}(0, z)=0, \lim _{\rho \rightarrow 0} f_{a}(\rho, z) / \rho^{2}>0$, with $a=2,3$. A semi-infinite spacelike rod corresponds to an axis of rotation, the associated coordinate being a rotation angle. Demanding regularity of the solutions at $\rho=0$ imposes a periodicity $2 \pi$ for both $\psi$ and $\varphi$. (However, when several $\psi$ - or $\varphi$-rods are present, it may be impossible to satisfy simultaneously all the periodicity conditions, see e.g. the examples in Appendix A).

One of the main advantages of this approach is that the topology of the horizon is automatically imposed by the rod structure. This provides a simple way to construct a variety of solutions with nontrivial topology of the horizon (including multi-black objects). 
Since (2.20) is linear, one can superpose different solutions for the same potential $U_{i}$. The nonlinear nature of the Einstein gravity manifests itself through the equation (2.4) for the metric function $\nu$.

\subsubsection{Higher dimensional structure}

The central point in this approach ${ }^{5}$ is that the rod structure, as defined above for the $d=5$ case, can be used also for $d>5$ solutions constructed within the ansatz (2.8). This fixes the boundary conditions along the $z$-axis for the functions $f_{i}$ and thus the topology of the horizon.

However, note that the interpretation of a rod as corresponding to a zero thickness source with linear mass density $1 / 2$, placed on the axis of symmetry in an auxiliary threedimensional flat space is no longer valid for $d>5$. Also, the relation (2.19) fails to be satisfied in this case, as one can see already for the simplest case of a Minkowski spacetime.

A crucial observation here is that, supposing the existence of a power series expansion in $\rho$, the Einstein equations imply ${ }^{6}$ the following form of the metric functions $f_{i}$ close to the $z$-axis, valid for any $d \geq 5$

$$
f_{i}(\rho, z)=f_{i 0}(z)+\rho^{2} f_{i 2}(z)+O\left(\rho^{3}\right),
$$

where $f_{i k}(z)$ are solutions of a complicated set of nonlinear second order ordinary differential equations which we shall not present here. Then, similar to the $d=5$ case, we suppose that the $z$-axis is divided into $N$ intervals-the rods of the solution. Except for the isolated points between the rods, one assumes that only one of the functions $f_{0}(0, z)$, $f_{2}(0, z), f_{3}(0, z)$, becomes zero for a given rod, while the remaining functions stay finite at $\rho=0$ in general. (In fact, if more than one of these functions is going to zero for a given $z$ inside a rod, one can prove following the arguments in 18 that we have a curvature singularity at that point.) Again, one imposes the condition that the $N$ intervals must add up to give an infinite rod.

A finite timelike rod corresponds to an event horizon, where ${ }^{7}$

$$
\begin{aligned}
& f_{0}(\rho, z)=\rho^{2} f_{02}(z)+\rho^{4} f_{04}(z)+\ldots, \quad f_{1}(\rho, z)=f_{10}(z)+\rho^{2} f_{12}(z)+\ldots \\
& f_{2}(\rho, z)=f_{20}(z)+\rho^{2} f_{22}(z)+\ldots, \quad f_{3}(\rho, z)=f_{30}(z)+\rho^{2} f_{32}(z)+\ldots
\end{aligned}
$$

with $\lim _{\rho \rightarrow 0} \rho^{2} f_{1} / f_{0}=c_{3}$. As we shall see in the next Subsection, this fixes the Hawking temperature of the solutions.

For a rod in the $\psi$-direction, one finds the following expansion of the metric functions as $\rho \rightarrow 0$ :

$$
\begin{aligned}
& f_{0}(\rho, z)=f_{00}(z)+\rho^{2} f_{02}(z)+\ldots, \quad f_{1}(\rho, z)=f_{10}(z)+\rho^{2} f_{12}(z)+\ldots \\
& f_{2}(\rho, z)=\rho^{2} f_{22}(z)+\rho^{4} f_{24}(z)+\ldots, \quad f_{3}(\rho, z)=f_{30}(z)+\rho^{2} f_{32}(z)+\ldots
\end{aligned}
$$

\footnotetext{
${ }^{5}$ Some aspects of the proposal in this work can be found also in Ref. [20], which considers the numerical construction of the five-dimensional black rings with two independent angular momenta.

${ }^{6} \mathrm{Such}$ an expansion is also required by the regularity of the Kretschmann scalar at $\rho=0$ (but it does not guarantee it automatically).

${ }^{7} f_{i k}(z)$ here should not be confused with those in (2.24).
} 
BH: $S^{d-2}$ horizon

(a)

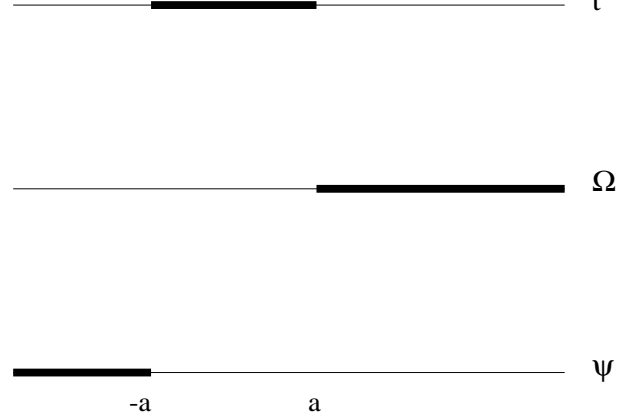

GBS: $\left(S^{2} \times S^{d-4}\right) \times S^{d-2}$ horizon

(c)
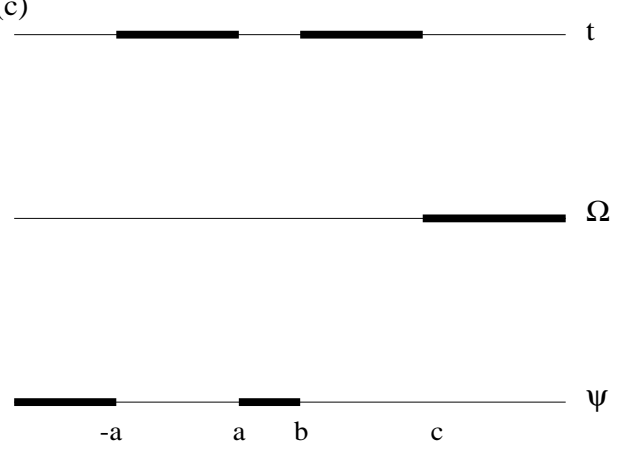

(b) GBR: $S^{2} \times S^{d-4}$ horizon $\mathrm{t}$ $\Omega$

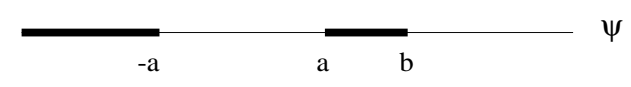

GBD: $\left(S^{2} \times S^{d-4}\right) \times\left(S^{2} \times S^{d-4}\right)$ horizon (d)

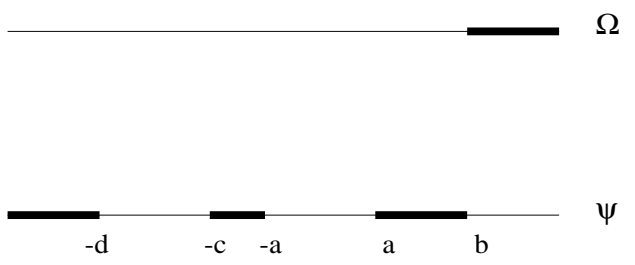

Figure 1. Rod structure of several static black objects in $d \geq 5$ spacetime dimensions. These include the Schwarzschild-Tangherlini black hole (BH), the generalized black ring (GBR), the generalized black Saturn (GBS) and the generalized black di-ring (GBD). In the diagrams the thin lines represent the $z$-axis and the thick lines denote the rods.

The important feature here is that the constraint equation $G_{\rho}^{z}=0$ implies $f_{10}(z) / f_{22}(z)=$ $c_{1}>0$, i.e. a well-defined periodicity for the coordinate $\psi$. The value of $c_{1}$ is not fixed apriori and follows from the details of the solutions.

For $d=5$, a similar result is found when interchanging $f_{2}$ and $f_{3}$, i.e. for a rod in the $\varphi$-direction. The periodicity of $\varphi$ there is arbitrary, being again fixed by the constraint equation $G_{\rho}^{z}=0$, i.e. $\lim _{\rho \rightarrow 0} \rho^{2} f_{1} / f_{3}=c_{2}>0$. However, the picture is very different ${ }^{8}$ for $d>5$, in which case we find

$$
\begin{aligned}
& f_{0}(\rho, z)=f_{00}(z)+\rho^{2} f_{02}(z)+\ldots, \quad f_{1}(\rho, z)=f_{10}(z)+\rho^{2} f_{12}(z)+\ldots \\
& f_{2}(\rho, z)=f_{20}(z)+\rho^{2} f_{22}(z)+\rho^{4} f_{24}(z)+\ldots, \quad f_{3}(\rho, z)=\rho^{2} f_{12}(z)+\ldots
\end{aligned}
$$

i.e. the Einstein equations impose the following requirement for an $\Omega-\operatorname{rod}: \lim _{\rho \rightarrow 0} \rho^{2} f_{1} / f_{3}=$ 1 , which is an important new feature. As we shall argue in the Section 2.5, this condition

\footnotetext{
${ }^{8}$ Formally, this is a consequence of the presence of the $(d-5)$ coefficients in the eqs. (2.3), (2.4).
} 
prevents us to construct $d>5$ static black rings (or multi-Schwarzschild-Tangherlini black holes) within the ansatz (2.8).

Thus, depending on the physical situation we consider, the boundary conditions along the $z$-axis are fixed by the above relations. The obvious boundary conditions for large $\rho,|z|$ are that $f_{i}$ approach the Minkowski background functions (which are read from 2.11):

$f_{0}(\rho, z)=1, \quad f_{1}(\rho, z)=\frac{1}{2 \sqrt{\rho^{2}+z^{2}}}, f_{2}(\rho, z)=\sqrt{\rho^{2}+z^{2}}+z, f_{3}(\rho, z)=\sqrt{\rho^{2}+z^{2}}-z$.

This is in fact the simplest solution of the Einstein equations in $d \geq 5$ dimensions, as we have seen already in the Section 2.2.1. There the function $f_{2}$ vanishes for $\rho=0, z<0$, and $f_{3}$ for $\rho=0, z>0$, which, in the language of the Weyl formalism, corresponds to two semi-infinite rods $[-\infty, 0]$ and $[0, \infty]$.

Similar to the $d=5$ case, the topology of an event horizon is fixed by the boundary conditions satisfied by $f_{2}$ and $f_{3}$ at the ends of the corresponding (finite) timelike rod. For example, if either end of this rod continues with rods of different directions $(\psi$ and $\Omega$ ), then the event horizon has an $S^{d-2}$ topology and (for a single horizon) the solution is a Schwarzschild-Tangherlini black hole (see Figure 1a). A black object with $S^{2} \times S^{d-4}$ topology of the horizon is a 'generalized black ring' and has the metric function $f_{2}$ vanishing at both ends of the finite timelike rod associated with the horizon, see Figure 1b. (For $d=5$, this corresponds to the static black ring in [2].) One can consider as well a 'generalized black Saturn' combining both types of black objects above, with two different horizons (Figure 1c). The rod structure for a solution consisting of two black holes, both of them with $S^{2} \times S^{d-4}$ topology of the horizon (thus a 'generalized black diring') is shown in Figure 1d. The basic properties of these objects are discussed in the next Section.

It is tempting to conjecture that, similar to the $d=5$ case [19], a $d>5$ solution within the ansatz (2.8), is uniquely specified by its rod structure.

\subsubsection{Physical quantities}

The ADM mass $M$ of the solutions ${ }^{9}$ can be read from the asymptotic expression for the metric function $g_{t t}$

$$
-g_{t t}=f_{0} \sim 1-\frac{16 \pi M}{(d-2) V_{d-2}\left(\rho^{2}+z^{2}\right)^{(d-3) / 4}}+\ldots .
$$

Supposing we have an event horizon at $\rho=0$ for some $z_{1} \leq z \leq z_{2}$, the horizon metric is given by ${ }^{10}$

$$
d \sigma^{2}=f_{1}(0, z) d z^{2}+f_{2}(0, z) d \psi^{2}+f_{3}(0, z) d \Omega_{d-4}^{2} .
$$

Two quantities associated with the event horizon are the event horizon area $A_{H}$ and the Hawking temperature. For the metric ansatz (2.8) these are given by

$$
A_{H}=\Delta \psi V_{d-4} \int_{z_{1}}^{z_{2}} d z \sqrt{f_{1}(0, z) f_{2}(0, z) f_{3}^{d-4}(0, z)}, \quad T_{H}=\frac{1}{2 \pi} \lim _{\rho \rightarrow 0} \sqrt{\frac{f_{0}(\rho, z)}{\rho^{2} f_{1}(\rho, z)}},
$$

\footnotetext{
${ }^{9}$ The discussion here follows the general formalism in [21], which contains also several applications for $d=4,5$ exact solutions.

${ }^{10}$ If there are several horizons, then one should write such an expansion for each of them.
} 
where $V_{d-4}$ is the area of the unit sphere $S^{d-4}$ and $\Delta \psi$ the periodicity of the angular coordinate $\psi$ on the horizon.

A solution with $n$ different event horizons satisfies the Smarr law

$$
M=\frac{d-2}{4(d-3)} \sum_{k=1}^{n} T_{H}^{(k)} A_{H}^{(k)} .
$$

Considering now the case of a space-like $\psi$-rod for some $z_{3} \leq z \leq z_{4}$, one starts by writing the line element on this $(d-2)$-dimensional surface $\Sigma$

$$
d \sigma^{2}=f_{1}(0, z) d z^{2}+f_{3}(0, z) d \Omega_{d-4}^{2}-f_{0}(0, z) d t^{2} .
$$

The first quantity of interest is the proper length of the rod

$$
L=\int_{z_{3}}^{z_{4}} d z \sqrt{f_{1}(0, z)}
$$

which, for a finite rod, differs from the coordinate distance $\Delta z=z_{4}-z_{3}$ (although it is proportional to it).

All solutions in this work possess a conical singularity for (at least) a region of the $z$-axis. To define a conical singularity for a rotational axis with angle $\psi$, one computes the proper circumference $C$ around the axis and its proper radius $R$ and defines

$$
\alpha=\left.\frac{d C}{d R}\right|_{R=0}=\lim _{\rho \rightarrow 0} \frac{\sqrt{g_{\psi \psi}} \Delta \psi}{\int_{0}^{\rho} \sqrt{g_{\rho \rho}} d \rho}=\lim _{\rho \rightarrow 0} \frac{\partial_{\rho} \sqrt{g_{\psi \psi}} \Delta \psi}{\sqrt{g_{\rho \rho}}},
$$

where $\Delta \psi$ is the period of $\psi$. The asymptotic flatness implies $\Delta \psi=2 \pi$. Then the presence of a conical singularity is now expressed ${ }^{11}$ by means of:

$$
\delta=2 \pi-\alpha=2 \pi\left(1-\lim _{\rho \rightarrow 0} \sqrt{\frac{f_{2}(\rho, z)}{\rho^{2} f_{1}(\rho, z)}}\right),
$$

such that $\delta>0$ corresponds to a conical deficit, while $\delta<0$ corresponds to a conical excess. A conical deficit can be interpreted as a 'string' stretched along on a certain segment of the $z$-axis, while a conical excess is a 'strut' pushing apart the rods connected to that segment (in fact, the 'struts' and 'strings' are $(d-3)$-dimensional surfaces, i.e. higher dimensional analogues of the $d=4$ cosmic strings). Also, a constant rescaling of $\psi$ can be used to eliminate possible conical singularities on a given segment, but in general, once this is fixed, there will remain conical singularities at other $\psi$-segments. For all solutions in this work, we have prefered to set the conical singularity on a finite $\psi$-rod such that our solutions are asymptotically flat, meaning that $\Delta \psi=2 \pi$. Moreover, in the presence of a conical singularity, the manifold $\mathcal{M}$ naturally factorizes as $\mathcal{M}=\mathcal{C}_{\alpha} \times \Sigma$, where $\mathcal{C}_{\alpha}$ is the two-dimensional conical surface $\rho-\psi$ and $\Sigma$ is the remaining $(d-2)$-dimensional surface, which may be seen as the world-volume of the conical defect [22].

\footnotetext{
${ }^{11}$ Note that, in some sence, fixing $\delta$ is the analogue of computing the Hawking temperature on the Euclidean section.
} 
For practical reasons, we have found it convenient to introduce the quantity

$$
\bar{\delta}=\frac{\delta /(2 \pi)}{1-\delta /(2 \pi)},
$$

which has a finite range $(\bar{\delta} \rightarrow-1$ for $\delta \rightarrow \pm \infty)$ and measures the 'relative angular deficit/excess'.

As argued in [21, 23], the asymptotically flat black objects with conical singularites still admit a thermodynamical description. Moreover, when working with the appropriate set of thermodynamical variables, the Bekenstein-Hawking law still holds for such solutions. The mass-energy which enters the first law of thermodynamics does not, however, coincide with the ADM mass; it differs from the latter by the energy associated with the conical singularity, as seen by an asymptotic, static observer.

The Ricci scalar of $\mathcal{M},{ }^{(\alpha)} R$, can be represented near $\Sigma$ in the following local form [22]:

$$
{ }^{(\alpha)} R=R+2(2 \pi-\alpha) \delta_{\Sigma}
$$

where $R$ is the curvature computed in the standard way on the smooth domain $\mathcal{M} / \Sigma$ of $\mathcal{M}$. Here, $\delta_{\Sigma}$ is the Dirac $\delta$-function, with $\int_{\mathcal{M}} f \delta_{\Sigma}=\int_{\Sigma} f$. A direct integration of (2.36) leads to [24]

$$
\int_{\mathcal{M}}{ }^{(\alpha)} R=\int_{\mathcal{M} / \Sigma} R+2(2 \pi-\alpha) \text { Area }
$$

where Area is the area of $\Sigma$, i.e. the space-time area of the conical singularity's worldvolume. For the metric ansatz in this work, the expression of the extensive parameter Area is

$$
\text { Area }=\beta V_{d-4} \int_{z_{3}}^{z_{4}} d z \sqrt{f_{0}(0, z) f_{1}(0, z) f_{3}^{d-4}(0, z)}
$$

$\beta=1 / T_{H}$ being the periodicity of the Euclidean time.

In the presence of conical singularities, the solutions cannot be viewed as vacuum solutions and there is a matter source (the struts) which supports the conical singularities. The stress energy tensor associated with the singularity can be computed by using the Einstein equations $G_{i j}=8 \pi T_{i j}$. The results in [22] also show that the singular part of the Ricci tensor has components only in the $\rho-\psi$ plane, such that $R_{i}^{j}=0$ for the remaining components. It follows that the only non-vanishing components of $T_{i}^{j}$ are

$$
T_{i}^{j}=-\delta_{i}^{j} \frac{1}{8 \pi}(2 \pi-\alpha) \delta_{\Sigma}, \quad \text { with }(i, j) \neq(\rho, \varphi) .
$$

A direct consequence of this result is that the conical deficit/excess as defined by (2.34), $\delta=2 \pi-\alpha$, corresponds to the pressure exerted by the strut. This is found by integrating the $T_{z}^{z}$-component over $\mathcal{C}_{\alpha}$

$$
P=\int_{\mathcal{C}_{\alpha}} T_{z}^{z}=-\frac{\delta}{8 \pi}
$$


Moreover, as seen from (2.39), the energy density $\mu$ of the matter source supporting the conical singularity is also $\mu=-\delta / 8 \pi$. Thus $\delta<0$ (the case of the solutions in this work) corresponds to a negative energy density source.

Another quantity of interest is the total energy associated with the strut as seen by a static observer placed at infinity. This is found by integrating the $T_{t}^{t}$-component over a $t=t_{0}=$ constant hyper-surface,

$$
E_{\text {int }}=-\int_{t=t_{0}} T_{t}^{t}=\frac{\delta}{8 \pi} \frac{\text { Area }}{\beta}=-P \mathcal{A}
$$

where we have defined

$$
\mathcal{A} \equiv \frac{\text { Area }}{\beta}
$$

Thermodynamics of a system with a conical singularity in the bulk can be approached ${ }^{12}$ by using the path-integral formulation of quantum gravity [13]. The first step here is to evaluate the total tree level Euclidean action of the system. The new feature introduced by the conical singularity is to add an extra contribution to this quantity which can be evaluated by using the relation (2.37). Then the total action is

$$
I=I_{0}-\frac{\delta}{8 \pi} \mathcal{A} \beta
$$

where $I_{0}$ is the usual tree level action [13] found when neglecting the conical singularity.

As argued in [21], the first law of thermodynamics for static vacuum solutions with a conical singularity reads

$$
d \mathcal{M}=T_{H} d S+\mathcal{T} d \mathcal{A},
$$

where $\mathcal{A}$ (as defined by (2.42) ) is the extensive parameter which takes into account the presence of the conical singularity and $\mathcal{T}=P=-\delta / 8 \pi$ is the associated "tension".

In a canonical ensemble, one keeps the Hawking temperature $T_{H}$ and the extensive parameter $\mathcal{A}$ fixed. The free energy $F$ is

$$
F\left[T_{H}, \mathcal{A}\right]=T_{H} I=\mathcal{M}-T_{H} S .
$$

Then the entropy $S$, mass $\mathcal{M}$ and tension $\mathcal{T}$ of the physical system are given by

$$
S=-\left.\frac{\partial F}{\partial T_{H}}\right|_{\mathcal{A}}, \quad \mathcal{M}=F+T_{H} S, \quad \mathcal{T}=\left.\frac{\partial F}{\partial \mathcal{A}}\right|_{T_{H}} .
$$

This approach has been applied in [21 for several $d=4,5$ static solutions with conical singularities in the bulk which are known in closed form, the effects of rotation being considered in [23]. The results there show that $S=A_{H} / 4$ in all cases, as expected.

In principle, one can use the same approach to discuss the thermodynamics of the solutions in this work. The only complication appears for multi-black objects. For thermal equilibrium, the individual black holes should have the same Hawking temperature. Moreover, if there are several different finite $\psi$-rods, then the corresponding tension parameters $\delta_{i}$ should be equal.

\footnotetext{
${ }^{12}$ In fact, it is possible to define the thermodynamic quantities also with the conical singularity stretching towards the boundary, see e.g. 25.
} 


\subsection{Numerical procedure}

All new solutions in this work are found within a nonperturbative approach by solving numerically a set of four nonlinear partial differential equations. These equations have dependence on two variables and are subject to suitable boundary conditions which follow from the required rod structure and asymptotic flatness.

In this scheme, the input parameters provided to the solver are the positions of the rods and the value $d$ of the spacetime dimension. The numerical integration eventually converges and provides an output consisting of the functions $f_{i}$ and their first and second derivatives with respect to $\rho$ and $z$. The relevant physical data (e.g. the ADM mass, the Hawking temperature(s) etc.) are computed from this numerical output.

In practice, we have found it convenient to take

$$
f_{i}=f_{i}^{0} F_{i}
$$

where $f_{i}^{0}$ are some background functions ${ }^{13}$, given by the metric functions of a $d=5$ solution with some rod structure. The numerical computation is performed working with the functions $F_{i}$. The advantage of this approach is that, since $F_{i}>0$ everywhere, the functions $f_{i}$ will automatically satisfy the desired rod structure also for the $d>5$ solutions. Moreover, this choice 'absorbes' the divergencies of the functions $f_{2}$ and $f_{3}$ as $\rho \rightarrow \infty$, $z \rightarrow \pm \infty$ originating in the imposed asymptotic behaviour (and also the divergencies of $f_{1}$ at the end of the finite $\operatorname{rods}^{14}$ ).

The equations satisfied by $F_{i}$ can easily be derived from those satisfied by $f_{i}$. As for the boundary conditions, the relations (2.24), (2.23) imply

$$
\left.\partial_{\rho} F_{i}\right|_{\rho=0}=0,
$$

for a $\psi$-rod or an event horizon, and

$$
\left.\partial_{\rho} F_{0}\right|_{\rho=0}=\left.\partial_{\rho} F_{1}\right|_{\rho=0}=\left.\partial_{\rho} F_{2}\right|_{\rho=0}=0,\left.\quad F_{1}\right|_{\rho=0}=\left.F_{3}\right|_{\rho=0},
$$

for an $\Omega$-rod. As $\rho \rightarrow \infty$ or $z \rightarrow \pm \infty$ one imposes the obvious condition $F_{i}=1$.

The constraint equation $G_{\rho}^{z}=0$ implies $F_{2} / F_{1}=$ const. on a $\psi$-rod. Now, to be consistent with the assumption of asymptotic flatness, one finds const. $=1$ for a $\psi$-rod extending to infinity. The value of this ratio for a finite rod results from the numerical results.

In the numerical calculations, one starts with the corresponding $d=5$ solution, (i.e. $F_{i}=1$ ), and slowly increases the parameter $d$. This leads to nontrivial deformations of $F_{i}$. The physical values of $d$ are integers. For all types of black objects in this work, we have studied solutions in $d=6$ dimensions in a systematic way. A number of $d=7$ solutions were also obtained but we did not fully investigate this case except for single black objects.

\footnotetext{
${ }^{13}$ Although sometimes it is not stated explicitly, the use of a suitable set of background functions is a common feature of numerical studies in general relativity. For example, for axisymmetric configurations, this factorizes the trivial angular dependence of the metric functions.

${ }^{14}$ Note that for all $d=5$ solutions the function $f_{1}(0, z)$ behaves as $1 /\left|z-z_{i}\right|$ as $z \rightarrow z_{i}$, with $z_{i}$ the end point of a finite rod. This behaviour is recovered for all $d>5$ solutions in this work.
} 
We think that solutions with $d>7$ are also very likely to exist; however, we could not construct them within the approach in this work and their study may require a different numerical method ${ }^{15}$.

In practice, one introduces also new compactified coordinates $x=\rho /(1+\rho), u=$ $\arctan (z)$, with $0 \leq x \leq 1,-\pi / 2 \leq u \leq \pi / 2$. The equations for $F_{i}$ are then discretized on a non-equidistant grid in $x$ and $u$. Typical grids used have sizes around $80 \times 150$, covering the full integration region. (See [29] and [30] for further details and examples for the numerical procedure.) All numerical calculations are performed by using the program FIDISOL/CADSOL [29], which uses a Newton-Raphson method ${ }^{16}$. This software provides also an error estimate for each unknown function. For $d=6$, the typical numerical error for the functions is estimated to be lower than $10^{-3}$. This error increases to several percent for most of the $d=7$ solutions.

Perhaps the most problematic aspect of this approach is that the derivatives of the functions $F_{i}$ diverge at the end points of the rods (although the functions $F_{i}$ are smooth everywhere). However, this appears already for the $d=5$ exact solutions ${ }^{17}$ and is an unavoidable feature of the Weyl-type coordinate system (2.8). To assure that these divergencies are coordinate artifacts, we have verified that the Kretschmann scalar stays finite everywhere, in particular at $\rho=0$ (here we ignore the $\delta$-Dirac terms in the expression of Riemann tensor in the presence of a conical singularity [22]). However, this non-smooth behaviour makes the numerics more involved as compared to other problems which were solved with similar methods and the same software. In particular, this approach requires a careful construction of the mesh in the region close to the end points of the rods. One should also remark that the presence of a conical singularity for the solutions in this work has a rather neutral effect on the numerics, since the solver does not notice it directly.

Furthermore, as a test of the software, we have recovered numerically the $d=6,7,8$ exact Schwarzschild-Tangherlini black hole starting with the $d=5$ solution, which, for the ansatz (2.8) possesses already the basic features of other more complex black objects. Another accuracy test of our solutions was provided by the Smarr relation (2.30).

A further numerical test is presented in Appendix $\mathrm{B}$, where the $d=5$ balanced black ring is recovered numerically. The background functions in this case correspond to those of the static exact solution.

\footnotetext{
${ }^{15}$ We believe this is not a worrisome aspect. For example, a similar situation was found in the past for nonuniform black strings in Kaluza-Klein theory, which at the beginning could be constructed numerically for $d=6$ only 14. However, subsequent work has managed to extend these solutions to $d=5$ [26] and $7 \leq d \leq 11$ [27]. Kaluza-Klein caged black holes were so far constructed within a nonperturbative approach for $d=5,6$ only 28 .

${ }^{16}$ Thus providing a good initial guess solution is essential for the convergence of the numerical process.

${ }^{17}$ For a $d=5$ black ring, the derivatives of the metric functions are finite everywhere when writing the solution in a ring-coordinate system. Although one can devise a ring-coordinate system (or more complicated versions adapted to the solutions in this work) for $d>5$ as well, we could not use it in practice within a numerical scheme. The main problem seems to be that the spacelike infinity is approached for a single point in that case.
} 


\subsection{The issue of $d>5$ black ring solutions}

One may expect that the approach proposed in this work can be used to construct a static black ring as well. But to be able to do so the metric has to have an unbroken $S O(d-3)$ symmetry group. The approximate solutions for thin black rings [9] do indeed preserve this symmetry and are cohomogenity-2. Therefore, we expect their static metrics in the $(\rho, z)$ coordinates to be within our ansatz.

The rod structure of these solutions in the generalized approach we are considering would consist of a semi-infinite space-like $\Omega-\operatorname{rod}[-\infty,-a]$ (with $f_{3}(0, z)=0$ there), a finite time-like rod $[-a, a]$ (the horizon, $f_{0}(0, z)=0$ ), a second (finite) $\Omega$-rod $[a, b]$ and a semi-infinite space-like $\psi$-rod $[b, \infty]$ (with a vanishing $f_{2}(0, z)$ ). Thus, it is obvious that the horizon topology of such an object would be $S^{d-3} \times S^{1}$.

In principle, one may consider as well multi-black objects with several $\Omega$-rods, the simplest case corresponding to a two Schwarzschild-Tangherlini black hole system, both with a $S^{d-2}$ topology of the horizon. Indeed, such solutions are known in closed form in four [31, 32], and five dimensions [33].

On general grounds, we know that higher dimensional black rings are possible and Ref. [9] found an approximate construction of such solutions based on the matched asymptotic expansion method. We note that the solutions in Ref. [9] describe balanced spinning black rings, but unbalanced and, in particular, static solutions should also exist. The analysis in Ref. [9] shows, that when the equilibrium condition is not satisfied, naked singularities arise in the plane of the ring. For higher-dimensional rings, the singularities are thus necessarily stronger than in five dimensions, where conical singularities suffice to balance the rings.

This observation is in correspondence with our analysis in (2.25), which shows that for $d>5$ one cannot assign to $\Omega_{d-4}$ any higher dimensional counterpart of a conical defect since $\lim _{\rho \rightarrow 0} \rho^{2} f_{3} / f_{1}=1$ in that case. One may conclude that the static black rings do not admit at $\rho=0$ a power series expansion of the form 2.22), since the static limit of a higher dimensional back ring will possess a stronger singularity than a conical one.

Clearly, for the envisaged numerical construction of such higher-dimensional static black rings the presence of curvature singularities provides a strong obstacle. ${ }^{18}$ Therefore we conclude that we cannot study $d$-dimensional static black rings within the metric (2.8) in a straightforward manner and a different approach seems to be required.

\footnotetext{
${ }^{18}$ Moreover, a $d$-dimensional static black ring might not be described within the ansatz 2.8 because the sphere $\Omega_{d-4}$ could be deformed in that case (i.e. the metric functions would have a dependence on (at least) one coordinate on that sphere).
} 

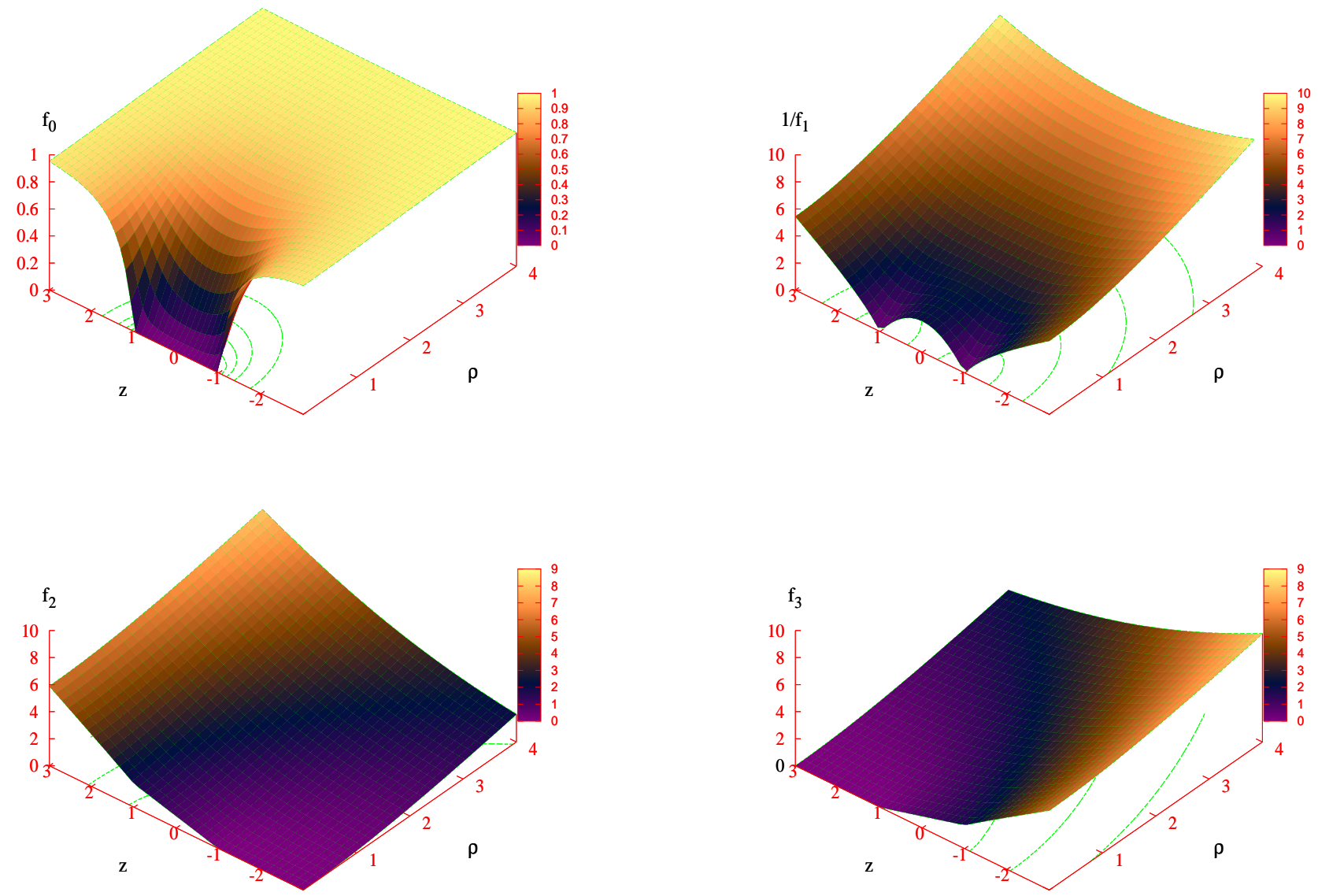

Figure 2. The profiles of the metric functions $f_{i}$ for a $d=8$ Schwarzschild-Tangherlini solution with $a=1$.

\section{General results for static black holes}

\subsection{Uni-horizon black holes}

\subsubsection{Schwarzschild-Tangherlini black hole: $S^{d-2}$ horizon}

The simplest example of a $d \geq 5$ black object that can be studied within this approach corresponds to the Schwarzschild-Tangherlini black hole. In Section 2.2.2 we have shown its form within the metric ansatz (2.8).

The rod structure of this black hole consists of (see Figure 1)

- A semi-infinite space-like $\operatorname{rod}[-\infty,-a]$ in the $\partial / \partial \psi$ direction (with $f_{2}(0, z)=0$ there),

- A finite time-like rod $[-a, a]$ in the $\partial / \partial t$ direction (the horizon, $\left.f_{0}(0, z)=0\right)$,

- A semi-infinite space-like $\operatorname{rod}[a, \infty]$ in the $\Omega$-direction (with a vanishing $f_{3}(0, z)$ ). 
Thus the topology of the horizon is $S^{d-2}$ as required. Requiring the absence of a conical singularity imposes a periodicity $2 \pi$ for the coordinate $\psi$. The only parameter here is $a>0$, which fixes the ADM mass of solutions (the only global charge for a SchwarzschildTangherlini black hole).

To get the feeling for the type of functions we will be dealing with later in the numerical computations, we show in Figure 2 the form of the metric components, namely the metric functions $f_{i}$, exhibiting this rod structure for a typical $d=8$ Schwarzschild-Tangherlini solution.

In principle, most of the physically relevant properties of the Schwarzschild-Tangherlini black hole can also be rederived within the metric ansatz (2.8). However, the required computation is much more difficult for that coordinate system.

\subsubsection{Generalized black ring: $S^{2} \times S^{d-4}$ horizon}

Very likely, these are the simplest $d>5$ black objects with a nonspherical topology of the horizon which can be constructed within a nonperturbative approach.

The rod structure in this case consists of (see Figure 1b)

- A semi-infinite space-like $\operatorname{rod}[-\infty,-a]$ in the $\partial / \partial \psi$ direction (with $f_{2}(0, z)=0$ there),

- A finite time-like rod $[-a, a]$ in the $\partial / \partial t$ direction (the horizon, $f_{0}(0, z)=0$ ),

- A a second (finite) $\psi$-rod $[a, b]\left(\right.$ with $\left.f_{2}(0, z)=0\right)$,

- A semi-infinite space-like rod $[b, \infty]$ in the $\Omega$-direction (with a vanishing $f_{3}(0, z)$ ).

The problem has two length scales $a$ and $b$, roughly corresponding to the event horizon radius and the radius of the round $\Omega_{d-4}$-sphere. These two input parameters fix the ADM mass $M$ and the tension $\delta$ which are the global charges here.

The basic properties of these solutions were discussed in [12 for $d=6,7$. For $d=5$, they correspond to the static black ring found in [2] (its basic properties are reviewed in Appendix A.1). The $d>5$ configurations share the basic properties of the five dimensional counterparts. This is why we propose to call them 'generalized black rings'.

Unfortunately, all static solutions with a $S^{2} \times S^{d-4}$ horizon topology have a conical excess $\delta$ on the finite $\psi$-rod. In terms of the dimensionless ratio $a / b$, the generalized black rings smoothly interpolate between two limits. First, as $a / b \rightarrow 1$, one finds $\bar{\delta} \rightarrow-1$ (i.e. the conical excess $\delta \rightarrow-\infty)$ and the Schwarzschild-Tangherlini metric is approached (the finite $\psi$-rod vanishes). As the second $\psi$-rod extends to infinity $(a / b \rightarrow 0)$, the radius on the horizon of the round $S^{d-4}$-sphere increases and asymptotically it becomes a $(d-4)$-plane, while $\bar{\delta} \rightarrow 0$. After a suitable rescaling, one finds the four dimensional Schwarzschild black hole uplifted to $d$ dimensions (i.e. a black $(d-4)$-brane). 

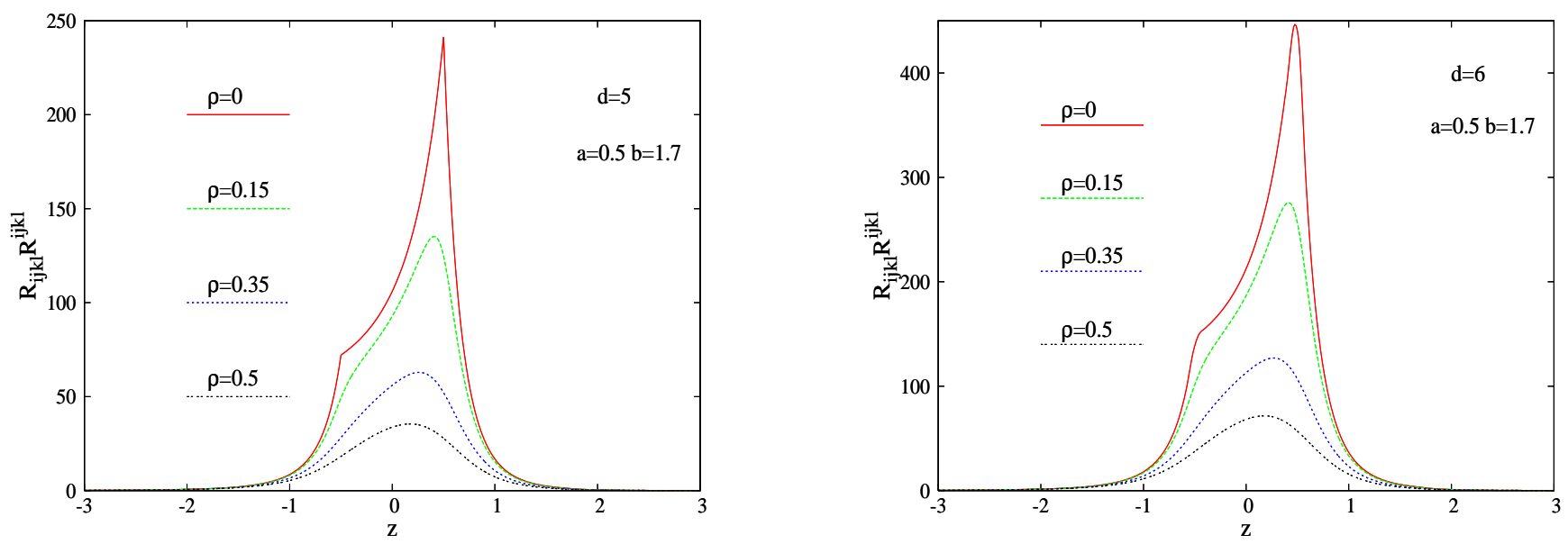

Figure 3. The Kretschmann scalar $R_{i j k l} R^{i j k l}$ is plotted as a function of $z$ for several values of $\rho$ for $d=5,6$ black objects with $S^{2} \times S^{d-4}$ event horizon topology.
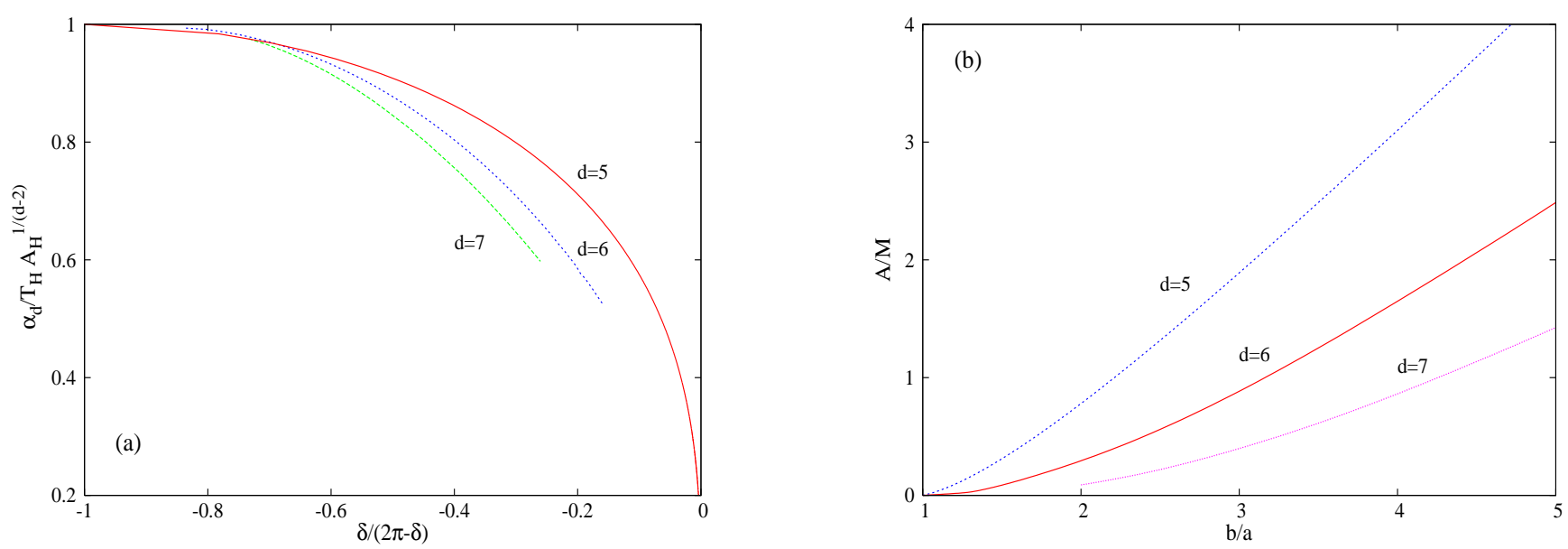

Figure 4. Features of generalized black ring solutions. (a): The scale free ratio $T_{H}^{-1} A_{H}^{1 /(d-2)}$ is shown as a function of the relative angular excess $\bar{\delta}=\delta /(2 \pi-\delta)$. The parameter $\alpha_{d}=(d-$ $3) 2^{(5-2 d) /(d-2)} \pi^{(3-d) / 2(d-2)} \Gamma((d-1) / 2)^{1 /(2-d)}$ has been chosen such that the point $(1,-1)$ on the plot corresponds to the Schwarzschild-Tangherlini black hole. (b): The scale free ratio $\mathcal{A} / \mathcal{M}$ is shown as a function of the ratio between the two length scales $b / a$.

The Kretschmann scalar $R_{i j k l} R^{i j k l}$ of typical $d=5,6$ solutions is shown in Figure 3. There one can see the nonsingular character of this type of configurations (here we do not consider the $\delta$-Dirac terms in the expression of Riemann tensor in the presence of a conical singularity [22]).

In Figure 4 we show the scale free ratio $T_{H}^{-1} A_{H}^{1 /(d-2)}$ as a function of the relative angular excess $\bar{\delta}=\delta /(2 \pi-\delta)$ for $d=5,6,7$ solutions. The dimensionless ratio $\mathcal{A} / \mathcal{M}$ is also shown there as a function of the ratio $b / a$ of the two length scales. One can see that, as expected, the pattern for $d=5$ repeats for the higher dimensional configurations. Further details on 
these solutions including typical profiles of the functions $f_{i}, F_{i}$ are given in Ref. [12].

\subsection{Multi-horizon black holes}

The general ansatz proposed in Section 2 offers the possibility to construct multi-black hole solutions as well. As discussed above, a limitation of this approach is that all configurations would have a single $\Omega$-rod extending to infinity. (For example, this prevents us from constructing $d>5$ multi-Schwarzschild-Tangherlini solutions.) However, the number of rods in the $\psi$ or $t$-directions are not constrained (although the numerical accuracy decreases with the number of rods).

In what follows, we present numerical evidence for the simplest asymptotically flat multi black objects, corresponding to 'generalized black Saturns' and 'generalized black dirings' (although more complex configurations are very likely to exist).

\subsubsection{Generalized black Saturn: $\left(S^{2} \times S^{d-4}\right) \times S^{d-2}$ horizon}

The $d=5$ static black Saturn describes a multi black hole configuration, with a black ring with horizon topology $S^{2} \times S^{1}$ around a Schwarzschild-Tangherlini black hole. This exact solution is found as a static limit of a rotating solution originally presented in [4] (see Appendix A.2 for a review of its basic properties).

We are interested in the generalization of this type of configuration to $d>5$ within the metric ansatz (2.8). There we have again two black objects, with a topology of the horizon $S^{2} \times S^{d-4}$ for the generalized black ring and $S^{d-2}$ for the central black hole. (Note that the horizon of the central black hole is not a round sphere).

This type of solution is found by imposing the following rod structure:

- A semi-infinite space-like $\operatorname{rod}[-\infty,-a]$ in the $\partial / \partial \psi$ direction (with $f_{2}(0, z)=0$ ),

- A finite time-like rod $[-a, a]$ in the $\partial / \partial t$ direction $\left(f_{0}(0, z)=0\right)$,

- A finite space-like rod $[a, b]$ in the $\partial / \partial \psi$ direction (where $f_{2}(0, z)=0$ again),

- A second finite time-like rod $[b, c]$ in the $\partial / \partial t$ direction, $\left(f_{0}(0, z)=0\right)$,

- A semi-infinite space-like $\operatorname{rod}[c, \infty]$ in the $\Omega$-direction (with a vanishing $f_{3}(0, z)$ ).

This rod structure is illustrated in Figure 1c.

The profiles of a tyical $d=7$ solution are shown in Figure 5 . Thus the problem has three input parameters $a, b, c$ fixing the mass of the individual components and the distance between them. In practice, one can always fix one of the parameters $a, b, c$ (i.e. the length scale of the problem) and vary the other two. 

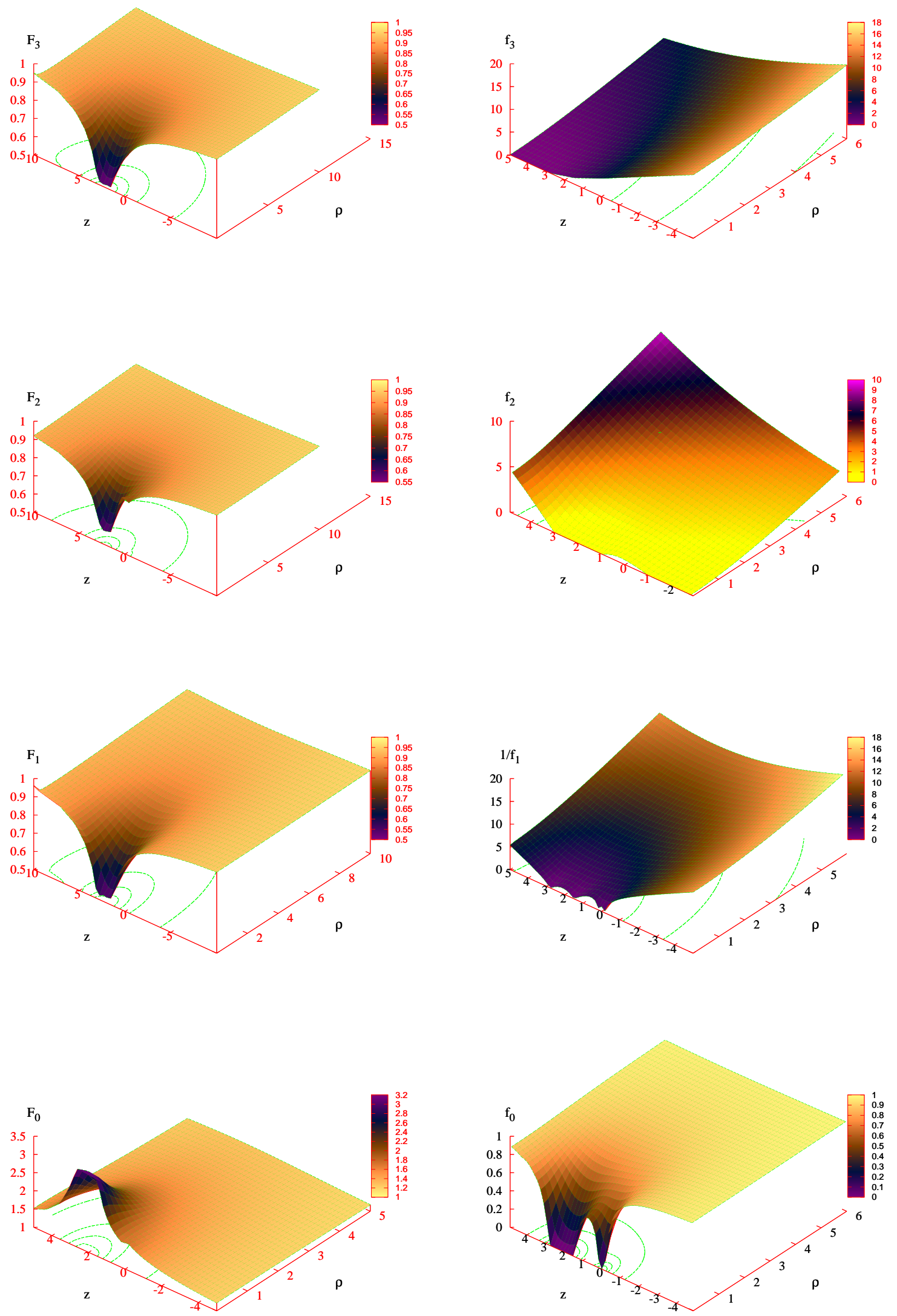

Figure 5. The profiles of the functions $F_{i}$ used in the numerical calculations and of the metric functions $f_{i}$ are shown for a typical $d=7$ black Saturn-type solution with $a=0.14, b=1.5$, $c=2.8$. 

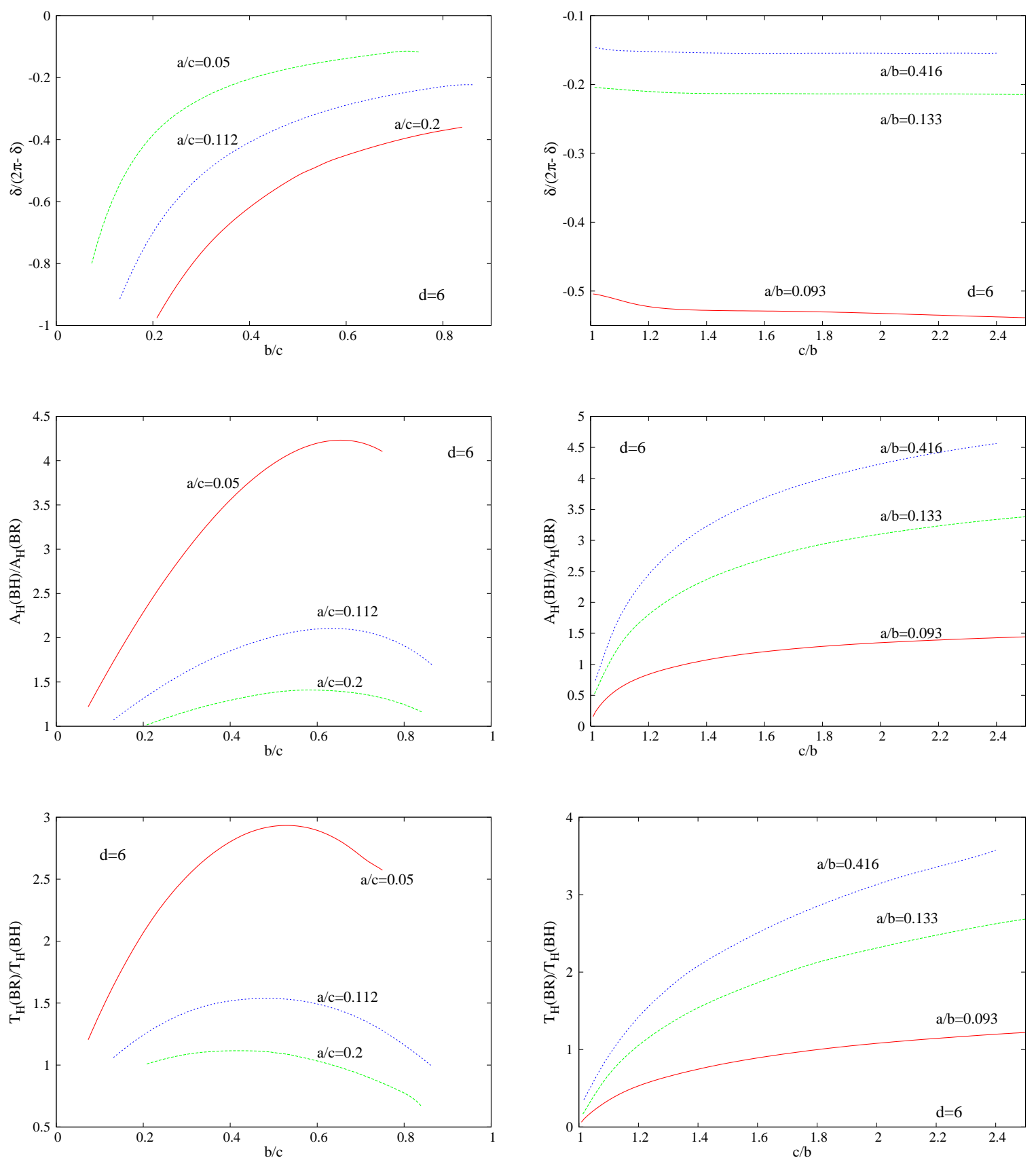

Figure 6. $d=6$ generalized black Saturn solutions: a number of relevant dimensionless quantities are shown as a function of the ratio $b / c$ for several fixed values of $a / c$ (left) and for a varying ratio $c / b$ for fixed $a / b$ (right). In these plots the indices BR, BH stand for the objects with $S^{2} \times S^{d-4}$ and $S^{d-2}$ horizon topology, respectively.

As expected, all our solutions have a conical singularity on the rod between the horizons, with $\delta<0$ there, which corresponds to a conical excess (and thus a negative energy density for the strut source). 
Starting with the dependence of solutions on the ratio $b / c$ for fixed $a / c$ one can see that the solutions smoothly interpolate between two limits. First, for $b / c \rightarrow 1$, the horizon with $S^{d-2}$ topology vanishes and the solution reduces to the higher dimensional generalizations [12] of the static black ring in [2]. Another limit of interest is $b / c \rightarrow a / c$, in which case the finite $\psi$-rod vanishes and a Schwarzschild-Tangherlini configuration is recovered.

We have studied as well the dependence of the solutions on the ratio $c / b$ for fixed $a / b$. There, for $c / b \rightarrow 1$, the horizon with $S^{d-2}$ topology vanishes and the solution reduces to a generalized black ring. Other interesting limits are $a \rightarrow b$ or $a=0$, in which cases the Schwarzschild-Tangherlini configuration is recovered.

Some results of the numerical integration supporting the above statements are shown in Figure 6 for several fixed values of $a / c$ and a varying $b / c$ (left) and for a varying ratio $c / b$ for fixed $a / b$ (right). One can see that the generic solutions are not in thermal equilibrium, $T_{H}^{B R} \neq T_{H}^{B H}$ (also, we could not find configurations with a vanishing Hawking temperature for one of the components). For all solutions, we have noticed a good qualitative agreement of their behaviour with that found for $d=5$ static black Saturns.

\subsubsection{Generalized di-rings: $\left(S^{2} \times S^{d-4}\right) \times\left(S^{2} \times S^{d-4}\right)$ horizon}

For $d=5$, the Einstein equations have an exact solution describing two concentric black rings [5], [6]. In the static limit, this asymptotically flat configuration is supported by a strut with positive pressure and negative energy density. The explicit form of the $d=5$ di-ring solution is given in Appendix A.3.

By using the same approach as in the previous cases, we could construct higher dimensional generalizations of this static configuration. There we have again two black objects, both with a topology of the horizon $S^{2} \times S^{d-4}$. They are found for the following rod structure:

- A semi-infinite space-like rod $[-\infty,-d]$ in the $\partial / \partial \psi$ direction $\left(\right.$ with $f_{2}(0, z)=0$ ),

- A finite time-like rod $[-d,-c]$ in the $\partial / \partial t$ direction $\left(f_{0}(0, z)=0\right)$,

- A finite time-like rod $[-c,-a]$ in the $\partial / \partial \psi$ direction (where $f_{2}(0, z)=0$ again),

- A second finite time-like rod $[-a, a]$ in the $\partial / \partial t$ direction $\left(f_{0}(0, z)=0\right)$,

- A finite space-like $\operatorname{rod}[a, b]$ in the $\partial / \partial \psi$ direction $\left(f_{2}(0, z)=0\right)$,

- A semi-infinite space-like $\operatorname{rod}[b, \infty]$ in the $\Omega$-direction (with a vanishing $f_{3}(0, z)$ ).

This rod structure is illustrated in Figure 1d.

Given the presence of four finite rods, finding such solutions is a more difficult problem, and we did not manage to obtain $d=7$ numerical solutions with reasonable accuracy. However, we think this is due to the limitations imposed by our approach only.

The metric functions $f_{i}$ and the functions $F_{i}$ used in the numerical calculations change smoothly with the rod parameters $a, b, c$ and $d$. Typical profiles of the solutions are presented in Figure 7 as a function of $z$ for several values of $\rho$. 

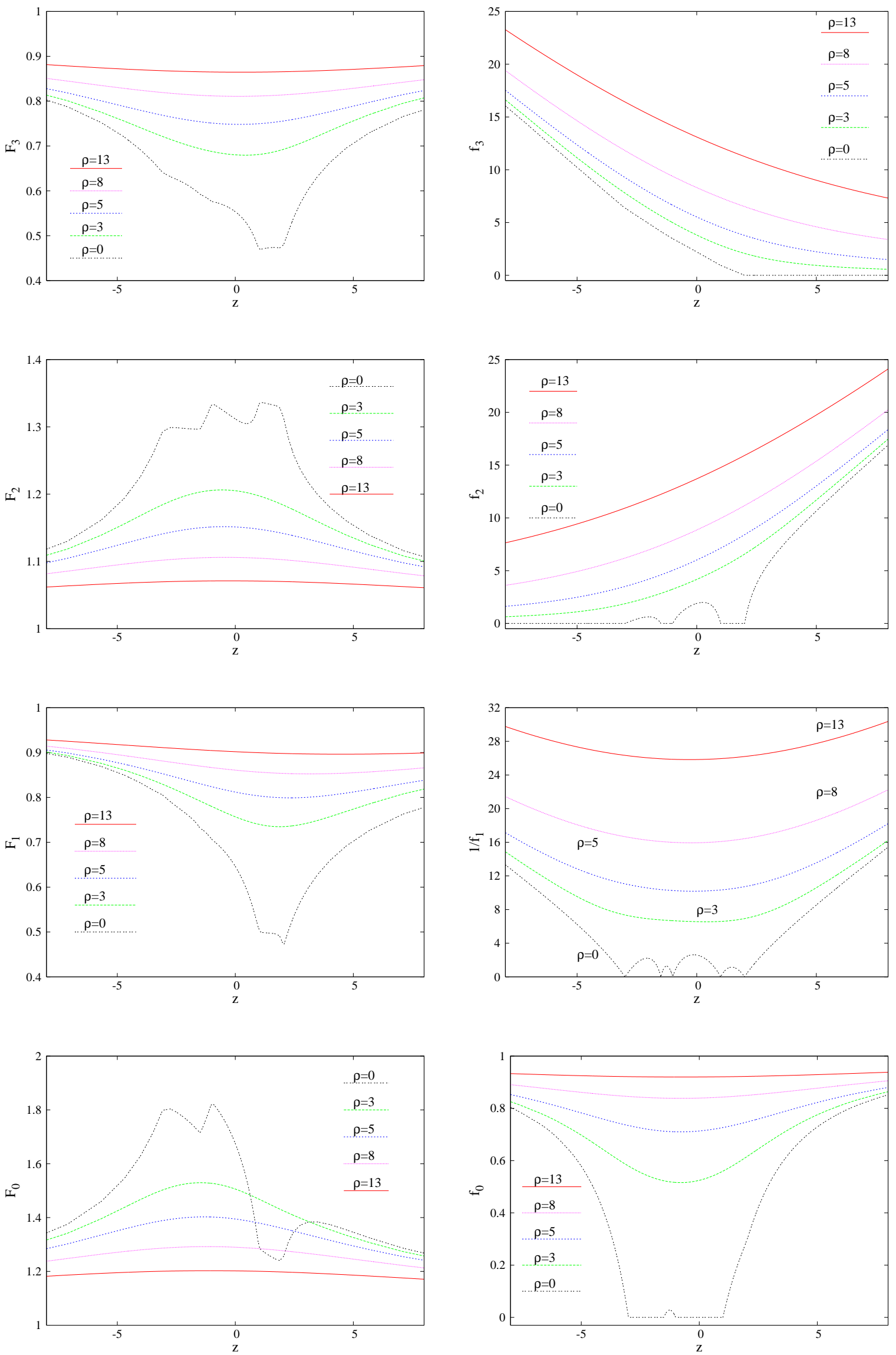

Figure 7. The profiles of the functions $F_{i}$ used in the numerical calculations and of the metric functions $f_{i}$ are shown for a typical $d=6$ generalized black diring solution with $a=1, b=2$, $c=1.5, d=3$. 

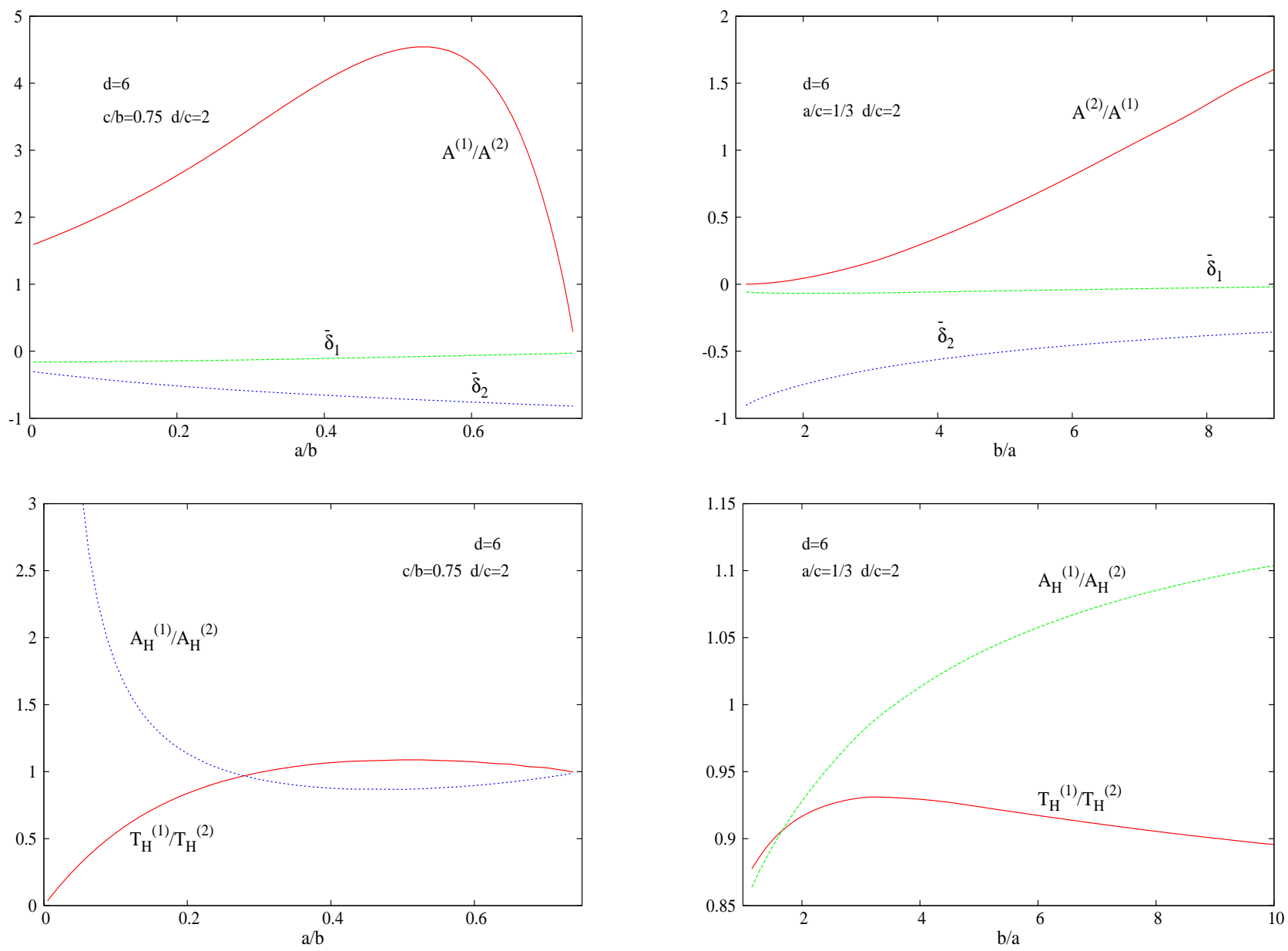

Figure 8. $d=6$ generalized black diring solutions: a number of relevant quantities are shown as a function of the ratio $a / b$ for several fixed values of $c / b, d / c$ (left) and for a varying ratio $b / a$ for fixed $a / c, d / c$ (right). In these plots the indices 1,2 stand for the objects with an event horizon at $\rho=0,-d \leq z \leq-c$ and $\rho=0,-a \leq z \leq a$, respectively.

One can see that the functions $F_{i}$ are smooth outside of the $z$-axis and show no sign of a singular behaviour (although they have a complicated behaviour at $\rho=0$ ).

In this case we have studied the dependence of the solutions on the parameters $a, b, c, d$ for two different situations (In fact a simple rescaling leads to a dependence on only three dimensionless quantities.). In the first case (see Figure 8 (left)), we have fixed $b, c, d$ and varied the parameter $a$ associated with the length of the second timelike rod (one of the constants $b, c, d$ can be taken to represent the length scale of the problem).

As $a \rightarrow 0$, a generalized black ring is approached since the second horizon vanishes, which implies $T_{H}^{(2)} \rightarrow \infty$ and $A_{H}^{(2)} \rightarrow 0$. As $a / c \rightarrow 1$ for fixed $b, c, d$, the first finite $\psi$-rod vanishes and the two horizons coalesce to form a single black object with $S^{2} \times S^{d-4}$ horizon topology.

The second case we have investigated corresponds to fixed $a, c, d$ and a varying $b$ (see Figure 8 (right)). The relevant limits here are $b / a \rightarrow 1$ and $b / a \rightarrow \infty$. As $a / b \rightarrow 1$, one finds 
$\bar{\delta}_{2} \rightarrow-1$ (i.e. the conical excess $\delta_{2} \rightarrow-\infty$ ) and a generalized black Saturn configuration is approached (the second finite $\psi-\operatorname{rod}$ vanishes). The case $b / a \rightarrow \infty$ is more involved and we have found it more difficult to investigate this limit. As the second $\psi$-rod extends to infinity, one expects to recover, after a suitable rescaling, the four dimensional doubleSchwarzschild configuration (i.e. the Bach-Weyl solution [31]) uplifted to six dimensions. This configuration still has a conical singularity in between the two black holes which provides the force balance that allows its existence.

Again, we have noticed a good qualitative agreement of this behaviour with that found for the $d=5$ static dirings exact solution (see Appendix A3). The generic solutions have

different Hawking temperatures for the individual components, $T_{H}^{(1)} \neq T_{H}^{(2)}$, and thus are not in thermal equilibrium. Also, it seems that there are no generalized black diring solutions with a vanishing Hawking temperature for one of the components.

\section{Remarks on rotating black holes with a nonspherical horizon topology}

All static solutions with a nonspherical horizon topology discussed above are plagued by conical singularities, which seems to be an unavoidable feature of all such asymptotically flat black objects.

However, for their $d=5$ counterparts, the conical singularities are eliminated by spinning the configurations, the rotation providing the centrifugal repulsion that allows a regular solution to exist.

On general grounds, one expects the $d>5$ new solutions in this work to possess rotating generalizations. Thus one may hope that by adding (at least) one spin to the system (without changing the rod structure) the configuration will be balanced (i.e. without conical singularities) for a critical value of the angular momentum. Unfortunately, there is no simple procedure to spin a given static system. Moreover, all known techniques fail for the solutions considered in this work. Therefore, we will again use a numerical approach.

In what follows, we present two different proposals for a metric ansatz which may describe rotating black holes with a nonspherical horizon topology. The first proposal is a straightforward extension of the ansatz (2.8) used in the static case, and leads to equations with dependence on two variables. The second ansatz proposal implies an $a$ priori dependence of the unknown metric functions on three variables.

\subsection{A generalized metric ansatz}

As discussed in [18], the Weyl coordinates and the rod-structure employed to construct $d=5$ static axisymmetric solutions can be generalized to the rotating case. Here we present arguments that the general framework proposed in Section 2 can also be generalized to include rotation.

In the simplest case, one considers a slightly more general metric form than (2.2), with

$$
d s^{2}=e^{2 \nu(\rho, z)}\left(d \rho^{2}+d z^{2}\right)+G_{i j}(\rho, z) d x^{i} d x^{j}+H(\rho, z) d \Omega_{d-4}^{2},
$$

and the coordinates $x^{i}=t, \psi$, which includes spinning configurations as well (since the metric component $g_{\psi t}$ can be nonzero). 
In what follows we show that, for $d>5$, a rotating black hole with a spherical topology of the horizon can be written within this ansatz (then it corresponds to the Myers-Perry solution with a single angular momentum). Another interesting case is represented by a black hole with a $S^{2} \times S^{d-4}$ topology of the horizon rotating with respect to the azimuthal angle $\psi$ (thus with a rotating $S^{2}$ ).

To make correspondence with (2.8), it is convenient to choose the following parametrization of (4.1)

$$
d s^{2}=-f_{0}(\rho, z) d t^{2}+f_{1}(\rho, z)\left(d \rho^{2}+d z^{2}\right)+f_{2}(\rho, z)(d \psi+W(\rho, z) d t)^{2}+f_{3}(\rho, z) d \Omega_{d-4}^{2} .
$$

The resulting equations for $f_{i}, W$ still have a dependence on only two variables, their structure being quite similar to that found in the static case (and thus we shall not write them here).

The rod structure as defined in Section 2 still holds for rotating solutions $\left(e . g \cdot f_{0}(0, z)=\right.$ 0 still defines the position of a horizon). Moreover, for a generic configuration, the metric functions $f_{i}$ admit the same expansion at $\rho=0$ as in the static case. As $\rho \rightarrow 0$, the new metric function $W(\rho, z)$ has the following form: $W(\rho, z)=w_{0}(z)+\rho^{2} w_{2}(z)+O\left(\rho^{4}\right)$, with $w_{0}(z)=\Omega_{H}=$ const. on a timelike rod, $\Omega_{H}$ being the event horizon velocity (note that the Killing vector $\partial / \partial t+\Omega_{H} \partial / \partial \psi$ is null at the horizon). At infinity one imposes again the same asymptotic behaviour for $f_{i}$, while $W \rightarrow 8 \pi J / V_{d-2}\left(\rho^{2}+z^{2}\right)^{(d-1) / 4}$, with $J$ the angular momentum of the solutions. The mass of the solutions is read again from the asymptotic expression of $f_{0}$.

However, other kinds of black holes, such as the spinning Myers-Perry black holes with multiple angular momenta, and presumably also the spinning balanced black holes with $S^{2} \times S^{2}$ horizon topology, do not fit within the conformal ansatz we use. For example, in the cases we studied, the conical sigularities of the spinning $S^{2} \times S^{2}$ black hole, could not be eliminated. This should not be taken as a sign of the absence of regular $S^{2} \times S^{2}$ black holes, but rather a consequence of the very restrictive form of the metric ansatz we employ.

Another argument comes from the recently found new extremal near horizon geometries in Ref. [34], that are similar to the near horizon geometries of the doubly equaly spinning extremal Myers-Perry black hole. These near horizon geometries were found as deformations of the doubly spinning extremal Myers-Perry black hole with equal spins and have angular cross terms that we do not consider in (4.1). So, taking into account the similarities between these near horizon geometries, we expect that balanced rotating $S^{2} \times S^{2}$ black hole or even more exotic, less symmetric, rotating black hole solutions not to fall in the (4.1) category.

\subsubsection{The singly spinning Myers-Perry black hole}

One can show that a Myers-Perry black hole with a single nonvanishing angular momentum can be written with the line element (4.1). This solution is usually written in the following form [3]

$$
\begin{aligned}
d s^{2}=-d t^{2}+\frac{\mu}{r^{d-5} \Sigma}(d t-a & \left.\sin ^{2} \theta d \psi\right)^{2}+\frac{\Sigma}{\Delta} d r^{2}+\Sigma d \theta^{2} \\
& +\left(r^{2}+a^{2}\right) \sin ^{2} \theta d \psi^{2}+r^{2} \cos ^{2} \theta d \Omega_{d-4}^{2},
\end{aligned}
$$


where

$$
\Sigma=r^{2}+a^{2} \cos ^{2} \theta, \quad \Delta=r^{2}+a^{2}-\frac{\mu}{r^{d-5}},
$$

such that at infinity the line element (2.9) is approached. Employing the same change of coordinates (2.13) as in the static case, where now

$$
G(r)=2 \int \frac{1}{\sqrt{\Delta}} d r
$$

we can get the metric to be in the form (4.1). Similar to the Schwarzschild-Tangherlini case, the above integral has a particularly simple form for $d=5^{19}$

$$
G(r)=2 \log \left[2\left(r+\sqrt{a+r^{2}-\mu}\right)\right],
$$

and for $d=7$, where

$$
G(r)=\log \left[a+2\left(r^{2}+\sqrt{a r^{2}+r^{4}-\mu}\right)\right] .
$$

Therefore the explicit form of the metric functions $\nu, G_{i j}$ and $H$ in (4.1) can be derived from (4.3). However, their expression is very complicated and we shall not present it here ${ }^{20}$.

One may wonder whether more general Myers-Perry solutions can also be written within the ansatz (4.1). For example, when $n \in N$ angular momenta are equal, the MyersPerry black hole exhibits a symmetry enhancement to $U(n) \times U(1) \times R_{t}$ symmetry. However, we have verified that such black holes require a more general metric form than (4.2).

\subsubsection{A rotating $S^{2}: d=6$ black holes with $S^{2} \times S^{2}$ topology of the horizon}

The Myers-Perry black hole has a $S^{d-2}$ horizon topology. However, in principle, solutions with a more complicated horizon topology can also be written within the ansatz (4.1).

For example, by employing the same methods as in the static case, we have constructed $d=6$ rotating black holes with a $S^{2} \times S^{2}$ topology of the event horizon (note that only one of these two spheres has a round shape). They are found by starting from a static solution and increasing the value of the angular velocity $\Omega_{H}$ of the event horizon, which enters the boundary conditions at $\rho=0$. Then, by varying also the second parameter $a / b$, associated with the position of the rods, the full set of 'generalized black rings' with a rotating $S^{2}$ can be explored in principle. As the second $\psi$-rod extends to infinity $(a / b \rightarrow 0)$, the radius of the horizon of the round $S^{2}$-sphere increases and asymptotically it becomes a two-plane. Here one expects to recover, after a suitable rescaling, the four dimensional Kerr black hole uplifted to six dimensions.

Although we did not yet investigate the full parameter space, all solutions we have constructed so far possess a conical singularity on the finite $\psi$-rod. This is not unexpected, since these configurations are natural higher dimensional counterparts of the $d=5$ black

\footnotetext{
${ }^{19}$ Note that also the expression for $\rho=\frac{1}{2} \sin 2 \theta\left(\frac{2 r \sqrt{a+r^{2}-\mu}}{a-\mu}\right), z=\frac{1}{2} \cos 2 \theta\left(1+\frac{2 r^{2}}{a-\mu}\right)$ are simple in this case.

${ }^{20}$ Their explicit form for $d=5$ is given e.g. in 18$]$.
} 
ring with a rotating $S^{2}$ found in [36]. Different from the balanced black ring in [1] (which has $g_{\psi t}=0, g_{\varphi t} \neq 0$ ), the solution in [36] has a conical singularity inside the ring for any allowed value of the angular momentum ${ }^{21}$. Balanced black rings with a rotating $S^{2}$ exist only if they rotate along $S^{1}$ as well [35]. Therefore, we expect a similar result also for $d>5$ solutions. However, this class of configurations would have a rotating $S^{d-4}$ and then would not be described by the ansatz (4.1).

A description of the spinning $d=6$ black holes with $S^{2} \times S^{2}$ topology of the horizon, together with generalizations for $d=7$ and multi-black hole objects will be presented elsewhere.

\subsection{A rotating $S^{d-4}$}

\subsubsection{A general metric ansatz}

Heuristically, to provide a centrifugal force which may balance a system with a nonspherical horizon topology, one needs to rotate the $S^{d-4}$ sphere $^{22}$. In principle, the simplest case corresponds to black objects with a single angular momentum with respect to a direction on $S^{d-4}$. For $d>5$, a possible generalization of the static ansatz (2.8) to this case has a dependence on $\rho, z$ and an angular variable $\theta$ on $S^{d-4}$, with

$$
d s^{2}=g_{i j}(\rho, z, \theta) d z^{i} d z^{j}+G_{i j}(\rho, z, \theta) d x^{i} d x^{j}+H(\rho, z, \theta) d \Omega_{d-6}^{2},
$$

where $z^{i}=\rho, z, \theta$ and $x^{i}=t, \psi, \varphi$ (note that the metric on $S^{d-4}$ is written in terms of a warped product of $S^{2}$ and $S^{d-6}$, with $d \Omega_{d-4}^{2}=d \theta^{2}+\sin ^{2} \theta d \varphi^{2}+\cos ^{2} \theta d \Omega_{d-6}^{2}$ in the absence of rotation).

An explicit parametrization of the above line element which makes contact with the static ansatz (2.8), proposing also a choice of the gauge in the $z^{i}$-sector, is

$$
\begin{aligned}
d s^{2}= & -f_{0}(\rho, z, \theta) d t^{2}+f_{1}(\rho, z, \theta)\left(d \rho^{2}+S_{1}(\rho, z, \theta) d z^{2}\right)+f_{2}(\rho, z, \theta) d \psi^{2} \\
& +f_{3}(\rho, z, \theta)\left(d \theta^{2}+S_{2}(\rho, z, \theta) \sin ^{2} \theta(d \varphi+W(\rho, z, \theta) d t)^{2}+S_{3}(\rho, z, \theta) \cos ^{2} \theta d \Omega_{d-6}^{2}\right),
\end{aligned}
$$

with $S_{i}=1$ and $W=0$ in the static case (note that $S_{3}=0$ for $d=6$; also we did not consider solutions with rotation on $S^{2}$, i.e. $\left.g_{\psi t}=0\right)$. Therefore finding rotating solutions with a nonspherical topology of the horizon reduces to solving a set of coupled partial differential equations for $f_{i}, S_{i}, W$, with suitable boundary conditions. However, this is a very difficult numerical problem, since the equations depend on three variables.

The only configurations we have attempted to construct within the above ansatz correspond to $d=6$ black holes with $S^{2} \times S^{2}$ topology of the horizon (this time both spheres deviate from sphericity). Again, one starts with static solutions and increases the value of the angular velocity $\Omega_{H}$. On general grounds, balanced solutions are expected to exist for a critical value of $\Omega_{H}$.

However, although in principle all methods and the software used in the static case can also be applied for this 3D problem, so far we could not make any progress in this direction.

\footnotetext{
${ }^{21}$ The thermodynamical properties of this solution are examined in 23].

${ }^{22}$ For example, in $d=5$ dimensions, the balanced ring is rotating with respect to the $S^{1}$ direction.
} 
The main problem is that we could not assure the convergence of the numerical process in the presence of rotation, even for small values of $\Omega_{H}$. This problem may be related to the issue of the gauge choice in this case ${ }^{23}$. For the version (4.9) of the generic ansatz (4.8), we have fixed the metric gauge by setting to zero the extradiagonal components $g_{\rho z}, g_{\rho \theta}, g_{z \theta}$. This seems to be a natural generalization of the "conformal gauge" employed in (2.8) (i.e. $g_{\rho \rho}=g_{z z}$ and $g_{\rho z}=0$ ), which is the most convenient choice in a numerical approach. However, different from the static case discussed above, we could not prove the consistency of the proposed metric ansatz (4.9). In other words, for rotating solutions there is no obvious way to prove that the constrained equations are solved automatically via Bianchi identities (plus suitable boundary conditions), as for the case of solutions with dependence on $\rho, z$ only (see the discussion in Section 2.1). This problem survives for other metric gauge choices we have considered.

One should also remark that $d>5$ balanced black rings with a single angular momentum can be constructed in principle by using the ansatz (4.9). However, all technical issues pointed out above apply for ring solutions as well. Moreover, higher dimensional black rings can also have a rotating $S^{2}$. Again, the exact solution is known only in five dimensions [35]. For $d>5$, we expect such solutions to have non trivial cross term metric components on the $S^{d-3}$ part. With the $S^{d-3}$-spins turned on, the solutions will presumably have $R \times U(1)^{n+1}$ symmetry and be cohomogeneity $d-(n+2)$ (with $n=[(d-2) / 2]$ ). And, even when $n$ angular momenta are set to be equal (and non-zero), the enhancement to a $U(n) \times U(1)$ rotational symmetry would not be enough to lead to a simple metric ansatz as it was the case for the Myers-Perry black hole with equal angular momenta (see e.g. [37]).

\subsection{2 $d=7$ rotating solutions with $S^{2} \times S^{3}$ horizon topology}

The only possibility we have found so far to construct nonperturbative spinning black objects with rotation on $S^{d-4}$ and possessing a nontrivial topology of the horizon, corresponds to the case $d-4=2 k+1$ (with $k=1,2, \ldots$ ). There the problem can be greatly simplified, when the a priori independent $(d-3) / 2$ angular momenta on $S^{d-4}$ are chosen to have equal magnitude, since this factorizes the angular dependence [37. The problem then reduces to studying the solutions of a set of five partial differential equations with dependence only on the variables $\rho, z$.

In what follows, we present some partial results for the simplest case $d=7$. The metric ansatz in this case is a straightforward reduction of (4.8), with

$$
\begin{aligned}
d s^{2}= & -f_{0}(\rho, z) d t^{2}+f_{1}(\rho, z)\left(d \rho^{2}+d z^{2}\right)+f_{2}(\rho, z) d \psi^{2}+f_{3}(\rho, z) d \theta^{2} \\
& +f_{4}(\rho, z)\left(\sin ^{2} \theta\left(d \varphi_{1}-W(\rho, z) d t\right)^{2}+\cos ^{2} \theta\left(d \varphi_{2}-W(\rho, z) d t\right)^{2}\right) \\
& -\left(f_{4}(\rho, z)-f_{3}(\rho, z)\right) \sin ^{2} \theta \cos ^{2} \theta\left(d \varphi_{1}-d \varphi_{2}\right)^{2}
\end{aligned}
$$

where $0 \leq \theta \leq \pi / 2,0 \leq \varphi_{1}, \varphi_{2} \leq 2 \pi$. The static ansatz (2.8) is recovered for $W=0$ and $f_{4}=f_{3}$.

\footnotetext{
${ }^{23}$ Note also that the complexity of the equations increases tremendously as compared to the static limit (2.3), 2.4).
} 
A suitable combination of the Einstein equations, $G_{t}^{t}=0, G_{\rho}^{\rho}+G_{z}^{z}=0, G_{\theta}^{\theta}=0$, $G_{\psi}^{\psi}=0, G_{\varphi_{1}}^{t}=0$ and $G_{\varphi_{1}}^{t}=0$, yields the following set of equations for the metric functions:

$$
\begin{aligned}
\nabla^{2} f_{0} & -\frac{1}{2 f_{0}}\left(\nabla f_{0}\right)^{2}+\frac{1}{2 f_{2}}\left(\nabla f_{0}\right) \cdot\left(\nabla f_{2}\right)+\frac{1}{f_{3}}\left(\nabla f_{0}\right) \cdot\left(\nabla f_{3}\right)+\frac{1}{2 f_{4}}\left(\nabla f_{0}\right) \cdot\left(\nabla f_{4}\right)-f_{4}(\nabla W)^{2}=0, \\
\nabla^{2} f_{1} & -\frac{1}{f_{1}}\left(\nabla f_{1}\right)^{2}-\frac{f_{1}}{2 f_{3}^{2}}\left(\nabla f_{3}\right)^{2}-\frac{f_{1} f_{4}}{2 f_{0}}(\nabla W)^{2}-\frac{f_{1}}{f_{0} f_{3}}\left(\nabla f_{0}\right) \cdot\left(\nabla f_{3}\right)-\frac{f_{1}}{f_{3} f_{4}}\left(\nabla f_{3}\right) \cdot\left(\nabla f_{4}\right) \\
& -\frac{f_{1}}{f_{2} f_{3}}\left(\nabla f_{2}\right) \cdot\left(\nabla f_{3}\right)-\frac{f_{1}}{2 f_{0} f_{2}}\left(\nabla f_{0}\right) \cdot\left(\nabla f_{2}\right)-\frac{f_{1}}{2 f_{0} f_{4}}\left(\nabla f_{0}\right) \cdot\left(\nabla f_{4}\right) \\
& -\frac{f_{1}}{2 f_{2} f_{4}}\left(\nabla f_{2}\right) \cdot\left(\nabla f_{4}\right)+\frac{2 f_{1}^{2}}{f_{3}^{2}}\left(4 f_{3}-f_{4}\right)=0, \\
\nabla^{2} f_{2}- & \frac{1}{2 f_{2}}\left(\nabla f_{2}\right)^{2}+\frac{1}{2 f_{0}}\left(\nabla f_{0}\right) \cdot\left(\nabla f_{2}\right)+\frac{1}{f_{3}}\left(\nabla f_{2}\right) \cdot\left(\nabla f_{3}\right)+\frac{1}{2 f_{4}}\left(\nabla f_{2}\right) \cdot\left(\nabla f_{4}\right)=0, \\
\nabla^{2} f_{3}+ & \frac{1}{2 f_{0}}\left(\nabla f_{0}\right) \cdot\left(\nabla f_{3}\right)+\frac{1}{2 f_{2}}\left(\nabla f_{2}\right) \cdot\left(\nabla f_{3}\right)+\frac{1}{2 f_{4}}\left(\nabla f_{3}\right) \cdot\left(\nabla f_{4}\right)+4 f_{1}\left(\frac{f_{4}}{f_{3}}-2\right)=0, \\
\nabla^{2} f_{4} & -\frac{1}{2 f_{4}}\left(\nabla f_{4}\right)^{2}+\frac{f_{4}^{2}}{f_{0}}(\nabla W)^{2}+\frac{1}{2 f_{0}}\left(\nabla f_{0}\right) \cdot\left(\nabla f_{4}\right)+\frac{1}{2 f_{2}}\left(\nabla f_{2}\right) \cdot\left(\nabla f_{4}\right) \\
& +\frac{1}{f_{3}}\left(\nabla f_{3}\right) \cdot\left(\nabla f_{4}\right)-\frac{4 f_{1} f_{4}^{2}}{f_{3}^{2}}=0, \\
\nabla^{2} W & -\frac{1}{2 f_{0}}\left(\nabla f_{0}\right) \cdot(\nabla W)+\frac{1}{2 f_{2}}\left(\nabla f_{2}\right) \cdot(\nabla W)+\frac{1}{f_{3}}\left(\nabla f_{3}\right) \cdot(\nabla W)+\frac{3}{2 f_{4}}\left(\nabla f_{4}\right) \cdot(\nabla W)=0 .
\end{aligned}
$$

All other Einstein equations except for $G_{\rho}^{z}=0$ and $G_{\rho}^{\rho}-G_{z}^{z}=0$ are linear combinations of those used to derive the above equations or are identically zero. Moreover, a similar reasoning to that presented for static solutions assures that the constraints $G_{\rho}^{z}=0$ and $G_{\rho}^{\rho}-G_{z}^{z}=0$ vanish identically via Bianchi identities plus suitable boundary conditions.

The only rotating solutions we have attempted to construct within this ansatz have an $S^{2} \times S^{3}$ topology of the horizon (i.e. $d=7$ generalized black rings) and a rod structure similar to that discussed in Section 2 (e.g. the horizon is located at $\rho=0$ and $-a \leq z \leq a$, where $\left.f_{0}=0\right)$. Moreover, the expansion at $\rho=0$ of the metric functions $f_{i}(i=0, \ldots, 3)$ is similar to that presented in the static case. For $\rho \rightarrow 0$ and $z \leq b$, the new functions $f_{4}, W$ have the following expansion

$$
f_{4}(\rho, z)=f_{40}(z)+\rho^{2} f_{42}(z)+\rho^{4} f_{44}(z)+\ldots, \quad W(\rho, z)=w_{0}(z)+\rho^{2} w_{2}(z)+\ldots,
$$

where $w_{0}(z)=$ const. for $-a \leq z \leq a$ (i.e. on the event horizon). The expansion of $f_{4}(\rho, z)$ is different for the $\Omega-\operatorname{rod}(i . e . \rho=0$ and a $z$-interval $[b, \infty])$, where $f_{4}(\rho, z)=$ $\rho^{2} f_{10}(z)+\rho^{4} f_{44}(z)+\ldots$ The obvious boundary conditions for large $\rho, z$ are that $f_{i}$ approach the Minkowski background functions 2.26), while $W=0$.

The Killing vector $\chi=\partial / \partial_{t}+\Omega_{1} \partial / \partial \varphi_{1}+\Omega_{2} \partial / \partial \varphi_{2}$ is orthogonal to and null on the horizon. For the solutions within the ansatz (2.8), the event horizon angular velocities are equal, $\Omega_{1}=\Omega_{2}=\left.W(0, z)\right|_{-a \leq z \leq a}=\Omega_{H}$.

As in the case of Myers-Perry black holes, these rotating black holes have an ergosurface inside of which observers cannot remain stationary, and will move in the direction of the rotation. 

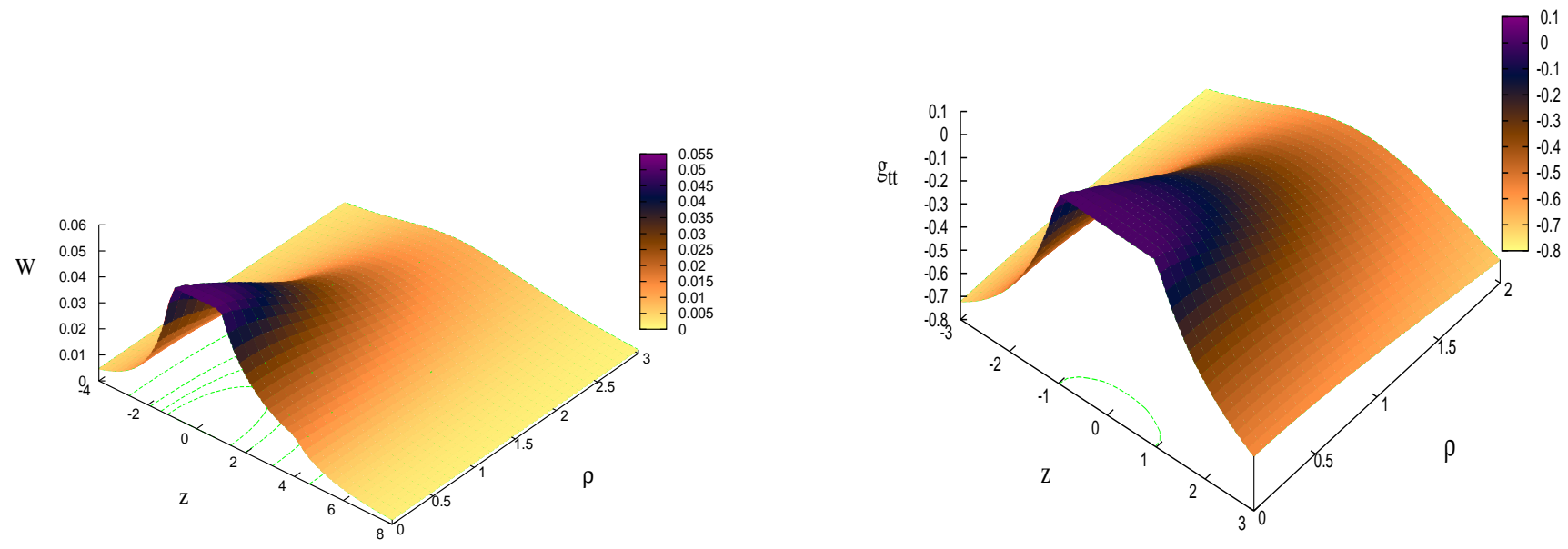

Figure 9. The metric function $W$ (left) and $g_{t t}=-f_{0}+f_{4} W^{2}$ (right) are shown close to the horizon for a $d=7$ rotating solution with $S^{2} \times S^{3}$ event horizon topology. The input parameters here are $a=1, b=4$ and $\Omega_{H}=0.05$. The contour line in the right panel indicates the ergo-region.

The ergosurface is located at $g_{t t}=0$, i.e.

$$
-f_{0}+f_{4} W^{2}=0
$$

and does not intersect the horizon.

The area $A_{H}$ and the Hawking temperature of the black hole can be expressed as

$$
A_{H}=\left.\frac{1}{2} \Delta \psi \int_{-a}^{a} d z f_{3} \sqrt{f_{1} f_{2} f_{4}}\right|_{\rho=0}, \quad T_{H}=\left.\frac{1}{2 \pi} \lim _{\rho \rightarrow 0} \sqrt{\frac{f_{0}(\rho, z)}{\rho^{2} f_{1}(\rho, z)}}\right|_{-a \leq z \leq a} .
$$

The ADM mass $M$ and the angular momenta $J_{1}=J_{2}=J$ of the solutions can be read from the asymptotic expression of $f_{0}, W$ :

$$
f_{0} \sim 1-\frac{16 \pi M}{5 V_{5}\left(\rho^{2}+z^{2}\right)}+\ldots, \quad W \sim \frac{8 \pi J}{V_{5}\left(\rho^{2}+z^{2}\right)^{3 / 2}} .
$$

These spinning black objects satisfy the Smarr formula

$$
\frac{4}{5} M=\frac{1}{4} T_{H} A_{H}+\frac{5}{2} \Omega_{H} J .
$$

The rotating solutions are found by starting with the static configurations with $S^{2} \times S^{3}$ topology of the horizon discussed in Section 3.1 .2 and increasing the horizon velocity $\Omega_{H}$. We have found that the absolute value of the conical excess $\delta$ decreases with $\Omega_{H}$, which 
suggests the existence of a critical value of the horizon angular velocity such that $\delta=0$ (this is the case for the general $d=5$ Emparan-Reall rotating black ring [18], see Appendix B). Unfortunately, the accuracy is lost and the numerical process diverges before approaching a balanced configuration, without being possible to identify a clear origin of this behaviour. However, a more sophisticated numerical approach may be able to find balanced black holes with $S^{2} \times S^{3}$ topology of the horizon.

As an example, we show in Figure 9 the metric function $W(\rho, z)$ and $g_{t t}(\rho, z)$ for the rotating solution with parameters $a=1, b=4$ and $\Omega_{H}=0.05$ (the shape of the other metric functions is similar to that found in the static case). In the right panel, one notices the existence of a region in the $(\rho, z)$ plane with $g_{t t}<0$ and of an ergosurface where $f_{0}=f_{4} W^{2}$.

\section{Conclusions}

The main purpose of this work was to present a general framework for the nonperturbative construction of a class of $d \geq 5$ static black objects with a nonspherical topology of the horizon. The solutions are found by solving numerically a set of four partial differential equations with suitable boundary conditions. Such an approach may be viewed as complementary to the approximate construction of such black objects developed recently in [9], [10], since it may work well if the length scales involved are not widely separated. Also, this made possible to consider some black object topologies that are not captured within the blackring/fold approach (e.g. $S^{2} \times S^{2}$ in $\left.d=6\right)$.

As a concrete application of the proposed formalism, we have presented numerical evidence for the existence of several $d>5$ black objects with a nonspherical topology of the horizon. These solutions represent generalizations of the $d=5$ static black rings, dirings and Saturns, with similar basic properties. Without entering into details, we mention that the double analytic continuation $\psi \rightarrow i T, t \rightarrow i \tau$ in the line element (2.8) leads to the more exotic interpretation of the solutions in this work as bubble-black hole sequences in a Kaluza-Klein theory. For example, the black hole with a $S^{2} \times S^{d-4}$ topology of the horizon becomes a pair of black objects (with one accelerated horizon) sitting on a bubble.

Not completely unexpected, our static solutions always possess conical singularities. The only way to achieve balance seems to be to rotate the solutions, no other mechanism being known at this moment. For example, the arguments in 12] put forward for generalized black rings apply directly to all static solutions in this work and one can show that the conical singularities plague also the Einstein-Maxwell-dilaton generalizations of the solutions in this paper ${ }^{24}$. Moreover, the results in [39] show that the Gauss-Bonnet corrections to Einstein gravity cannot eliminate the conical singularity of a $d=5$ static black ring, and we expect a similar result to hold also for the higher dimensional configurations discussed in this work.

\footnotetext{
${ }^{24}$ Static balanced black objects with a nonspherical topology of the horizon may exist, however, if the gauge fields are not vanishing at infinity, i.e. for an asymptotic Melvin structure of spacetime [12]. Such solutions are known in closed form in $d=5$ dimensions, see e.g. [38].
} 
As argued in Section 4, the construction of the rotating balanced version of the solutions we have considered is a much more difficult task. However, based on the experience with $d=$ 5 exact solutions, it is likely that some of the qualitative features of the static configurations will hold also in the spinning case.

A further generalization of the solutions may be along the lines of Ref. [40], where (apparent) horizons of topology $S^{n} \times S^{m+1}, n, m \geq 1$ were considered.

Thus, we expect that the new configurations discussed in this work represent just 'the tip of the iceberg' and a variety of new $d>5$ black objects with nonspherical topology of the horizon are likely to be discovered within a nonperturbative approach. In any such attempt, the rod structure of the solutions (or a suitable generalization of it) would represent an important ingredient, as a tool to fix the topology of the horizon. For example, it would be interesting to adapt the numerical methods in this work for the domain structure approach introduced recently in [17].

In our opinion, any progress in this direction would require the development of a consistent numerical scheme capable to solve as a boundary value problem the Einstein equations with a dependence on at least three coordinates.

\section{Acknowledgements}

We are grateful to Roberto Emparan for clarifying comments on the singularity structure of $d>5$-dimensional black rings, and for bringing Ref. [40] to our attention, pointing out a possible extension of our current results. M.J.R. wants to thank Oscar Varela for helpful discussions. B.K. gratefully acknowledges support by the DFG. The work of E.R. was supported by a fellowship from the Alexander von Humboldt Foundation and the Science Foundation Ireland (SFI) project RFP07-330PHY.

\section{A. Five-dimensional seeds}

For completeness and comparison with the higher dimensional counterparts, we present in what follows the expression (in a form suitable for numerical calculations) and some basic properties of the five dimensional seed solutions ${ }^{25}$. Also, the functions $f_{i}$ below are used as background functions for the corresponding higher dimensional solutions.

Moreover, based on the results below, one can easily construct e.g. the $d=5$ counterparts of the Figures 6 and 8 , which clearly show that the $d=5$ pattern repeats in higher dimensions.

\section{A.1 The static black ring}

The metric functions $f_{i}$ of the static black ring are given by [2], [18]

$$
\begin{gathered}
f_{0}=\frac{P_{2}+2 \xi_{2}}{P_{1}+2 \xi_{1}}, f_{1}=\frac{\left(P_{1}+2 \xi_{1}+P_{2}\right)\left(P_{1}+P_{2}+\xi_{1}+\xi_{2}+c\left(-\xi_{1}+\xi_{2}+2 \xi_{3}-P_{1}+P_{2}+2 P_{3}\right)\right.}{8(1+c)\left(P_{1}+\xi_{1}\right)\left(P_{2}+\xi_{2}\right)\left(P_{3}+\xi_{3}\right)}, \\
f_{2}=\frac{P_{2}}{P_{1}}\left(P_{3}+2 \xi_{3}\right), \quad f_{3}=P_{3},
\end{gathered}
$$

\footnotetext{
${ }^{25}$ To the best of our knowledge, this study is missing in the literature and thus may be useful for future studies.
} 
where $\xi_{i}=z-z_{i}$

$$
P_{i}=\sqrt{\rho^{2}+\left(z-z_{i}\right)^{2}}-\left(z-z_{i}\right)
$$

and

$$
z_{1}=-a, \quad z_{2}=a, \quad z_{3}=b,
$$

$a$ and $b$ being two positive constants, with $a<b$ and $c=a / b$.

The leading order expansion as $\rho \rightarrow 0$ of these functions is:

$$
\begin{aligned}
& f_{0}=\frac{z+a}{z-a}, \quad f_{1}=-\frac{a-z}{2(b-z)(a+z)}, \quad f_{2}=\frac{\rho^{2}(z-a)}{2(b-z)(a+z)}, \\
& f_{3}=2(b-z), \quad \text { for } \quad-\infty<z \leq-a, \\
& f_{0}=\frac{\rho^{2}}{4\left(a^{2}-z^{2}\right)}, \quad f_{1}=\frac{2 a^{2}}{(a+b)\left(a^{2}-z^{2}\right)}, \quad f_{2}=\frac{2\left(a^{2}-z^{2}\right)}{(b-z)}, \\
& f_{0}=1-\frac{2 a}{z+a}, \quad f_{1}=\frac{(b-a)(z+a)}{2(b+a)(z-a)(b-z)}, \quad f_{2}=\frac{\rho^{2}(z+a)}{2(z-a)(b-z)}, \\
& f_{0}=1-\frac{2 a}{z+a}, \quad f_{1}=\frac{1}{2(z-b)}, \quad f_{2}=\frac{2(z-b)(z+a)}{(z-a)}, \quad f_{3}=\frac{\rho^{2}}{2(z-b)}, \quad \text { for } \quad b \leq z<\infty .
\end{aligned}
$$

The mass, event horizon area and temperature of the $d=5$ static black ring are:

$$
M^{(5)}=\frac{3 a V_{2}}{4 \pi}, \quad A_{H}^{(5)}=8 a^{2} \sqrt{\frac{2}{a+b}} V_{2}, \quad T_{H}^{(5)}=\frac{1}{4 \pi a} \sqrt{\frac{a+b}{2}} .
$$

These black rings have a conical deficit for the finite $\psi$-rod, with

$$
\delta=2 \pi\left(1-\sqrt{\frac{b+a}{b-a}}\right) .
$$

\section{A.2 The static Saturn}

The metric functions are given in this case by [4]

$$
\begin{aligned}
& f_{0}=\frac{P_{1} P_{3}}{P_{2} P_{4}}, \quad f_{1}=\frac{\left(\rho^{2}+P_{1} P_{2}\right)^{2}\left(\rho^{2}+P_{2} P_{3}\right)^{2}\left(\rho^{2}+P_{1} P_{4}\right)\left(\rho^{2}+P_{3} P_{4}\right) P_{4}}{\left(\rho^{2}+P_{1}^{2}\right)\left(\rho^{2}+P_{2}^{2}\right)\left(\rho^{2}+P_{3}^{2}\right)\left(\rho^{2}+P_{4}^{2}\right)\left(\rho^{2}+P_{1} P_{3}\right)^{2}\left(\rho^{2}+P_{2} P_{4}\right)}, \\
& f_{2}=\frac{\rho^{2} P_{2}}{P_{1} P_{3}}, \quad f_{3}=P_{4},
\end{aligned}
$$

where $P_{i}$ is given by $(\mathrm{A} .2)$ and

$$
z_{1}=-a, \quad z_{2}=a, \quad z_{3}=b, \quad z_{4}=c
$$

$a, b$ and $c$ being three positive constant, with $a \leq b \leq c$. The notation here is somehow arbitrary and has been chosen to make contact with the results in [12]. 
This describes a multi-black hole solution, with a black ring with horizon topology $S^{2} \times S^{1}$ around an $S^{3}$ black hole.

The leading order expansion as $\rho \rightarrow 0$ of these functions is:

$$
\begin{aligned}
& f_{0}=\frac{(z+a)(z-b)}{(a-z)(c-z)}, f_{1}=\frac{z-a}{2(b-z)(a+z)}, f_{2}=\frac{\rho^{2}(z-a)}{2(b-z)(a+z)}, \\
& f_{3}=2(c-z) \text {, for }-\infty<z \leq-a, \\
& f_{0}=\frac{\rho^{2}(b-z)}{4(c-z)\left(a^{2}-z^{2}\right)}, f_{1}=\frac{2 a^{2}(a+c)(b-z)}{(a+b)^{2}(c-z)\left(a^{2}-z^{2}\right)}, f_{2}=\frac{2\left(a^{2}-z^{2}\right)}{(b-z)} \\
& f_{3}=2(c-z) \text {, for }-a \leq z \leq a, \\
& f_{0}=\frac{(a-z)(z-b)}{(c-z)(z+a)}, f_{1}=\frac{(a-b)^{2}(a+c)(a+z)}{2(a+b)^{2}(a-c)(a-z)(b-z)}, \\
& f_{2}=\frac{\rho^{2}(z+a)}{2(a-z)(z-b)}, f_{3}=2(c-z), \text { for } a \leq z \leq b, \\
& f_{0}=\frac{(a-z) \rho^{2}}{4(b-z)(c-z)(z+a)}, f_{1}=\frac{(a+c)(a-z)}{2(a-c)(b-z)(a+z)}, \\
& f_{2}=\frac{2(a+z)(z-b)}{(z-a)}, f_{3}=2(c-z), \text { for } b \leq z \leq c \\
& f_{0}=\frac{(a-z)(c-z)}{(z+a)(z-b)}, f_{1}=\frac{1}{2(z-c)}, f_{2}=\frac{2(b-z)(z+a)}{(a-z)}, f_{3}=\frac{\rho^{2}}{2(z-c)}, \text { for } c \leq z<\infty .
\end{aligned}
$$

The event horizon area and temperature of the black hole with horizon topology $S^{2} \times S^{1}$ are:

$$
A_{B R}^{(5)}=4 \pi^{2} \frac{4 \sqrt{2} a^{2} \sqrt{a+c}}{a+b}, \quad T_{B R}^{(5)}=\frac{a+b}{4 \sqrt{2} \pi a \sqrt{a+c}} .
$$

The same quantities for the black hole with horizon topology $S^{3}$ are:

$$
A_{S T}^{(5)}=4 \pi^{2} \sqrt{2}(c-b) \sqrt{\frac{(a+c)(c-b)}{c-a}}, \quad T_{S T}^{(5)}=\frac{1}{\sqrt{2} \sqrt{2} \pi} \sqrt{\frac{c-a}{(a+c)(c-b)}} .
$$

These solutions have a conical deficit for the finite $\psi$-rod (i.e. $\rho=0,-a \leq z \leq a$ ), with

$$
\delta=2 \pi\left(1-\frac{b+a}{b-a} \sqrt{\frac{c-a}{c+a}}\right),
$$

which prevents the configuration from collapsing.

The ADM mass of this system, as measured at infinity is:

$$
M=\frac{3 \pi}{4}(2 a-b+c) .
$$

\section{A.3 The static di-ring}

The metric functions are given in this case by

$$
\begin{gathered}
f_{0}=\frac{P_{1} P_{3}}{P_{2} P_{4}}, \quad f_{2}=\frac{\rho^{2} P_{2} P_{4}}{P_{1} P_{3} P_{5}}, \quad f_{3}=P_{5} \\
f_{1}=\frac{P_{5}\left(\rho^{2}+P_{1} P_{2}\right)^{2}\left(\rho^{2}+P_{1} P_{3}\right)^{2}\left(\rho^{2}+P_{1} P_{4}\right)^{2}\left(\rho^{2}+P_{3} P_{4}\right)^{2}\left(\rho^{2}+P_{2} P_{5}\right)\left(\rho^{2}+P_{4} P_{5}\right)}{\left(\rho^{2}+P_{1}^{2}\right)\left(\rho^{2}+P_{2}^{2}\right)\left(\rho^{2}+P_{3}^{2}\right)\left(\rho^{2}+P_{4}^{2}\right)\left(\rho^{2}+P_{5}^{2}\right)\left(\rho^{2}+P_{1} P_{3}\right)^{2}\left(\rho^{2}+P_{2} P_{4}\right)^{2}\left(\rho^{2}+P_{1} P_{5}\right)\left(\rho^{2}+P_{3} P_{5}\right)}
\end{gathered}
$$


where $P_{i}$ is still given by $($ A.2 and

$$
z_{1}=-d, \quad z_{2}=-c, \quad z_{3}=-a, \quad z_{4}=a, \quad z_{5}=b,
$$

$a, b, c$ and $d$ being three positive constants, with $d>c>a$ and $a \leq b$. This describes a configuration consisting of two concentric black rings with horizon topology $S^{2} \times S^{1}$.

The leading order expansion as $\rho \rightarrow 0$ of the metric functions is:

$$
\begin{aligned}
& f_{0}=\frac{(z+a)(d+z)}{(z-a)(c+z)}, f_{1}=\frac{(a-z)(c+z)}{2(a+z)(z-b)(d+z)}, \\
& f_{2}=\frac{\rho^{2}(a-z)(c+z)}{2(a+z)(z-b)(d+z)}, f_{3}=2(b-z), \text { for }-\infty<z \leq-d, \\
& f_{0}=\frac{\rho^{2}(a+z)}{4(a-z)(c+z)(d+z)}, f_{1}=\frac{(c-d)^{2}(a+d)^{2}}{2(a-d)^{2}(b+d)} \frac{(a+z)}{(a-z)(c+z)(d+z)}, \\
& f_{2}=\frac{2(a-z)(c+z)(d+z)}{(b-z)(a+z)}, f_{3}=2(b-z), \text { for }-d \leq z \leq-c, \\
& f_{0}=\frac{(a+z)(c+z)}{(z-a)(z+d)}, f_{1}=\frac{(a+d)^{2}}{2(a-d)^{2}(b+d)} \frac{(a+z)(d+z)}{(a-z)(c+z)}, \\
& f_{2}=\frac{\rho^{2}(a-z)(d+z)}{2(a+z)(z-b)(c+z)}, f_{3}=2(b-z) \text {, for }-c \leq z \leq-a, \\
& f_{0}=\frac{(c+z) \rho^{2}}{4(d+z)\left(a^{2}-z^{2}\right)}, f_{1}=\frac{(b+c)(a+d)^{2}}{2(a+c)^{2}(b+d)} \frac{(a-z)(c+z)}{(a+z)(z-b)(d+z)}, \\
& f_{2}=\frac{2\left(a^{2}-z^{2}\right)(d+z)}{(b-z)(c+z)}, f_{3}=2(b-z), \text { for }-a \leq z \leq a, \\
& f_{0}=\frac{(c+z)(z-a)}{(z+a)(z+d)}, f_{1}=\frac{(b+c)(a+z)(d+z)}{2(a+b)(b+d)(z-a)(c+z)}, \\
& f_{2}=\frac{\rho^{2}(a+z)(d+z)}{2(a-z)(z-b)(c+z)}, f_{3}=2(b-z), \text { for } a \leq z<b, \\
& f_{0}=\frac{(c+z)(z-a)}{(z+a)(z+d)}, f_{1}=\frac{1}{2(z-b)} \\
& f_{2}=\frac{2(a+z)(z-b)(d+z)}{(z-a)(c+z)}, f_{3}=\frac{\rho^{2}}{2(z-b)}, \text { for } b \leq z<\infty \text {. }
\end{aligned}
$$

The event horizon area and temperature of the "left" black ring (with the horizon located at $\rho=0,-d \leq z \leq-c)$ are:

$$
A_{L}^{(5)}=4 \pi^{2} \frac{\sqrt{2}(c-d)^{2}(a+d)}{(d-a)} \frac{1}{\sqrt{b+d}}, \quad T_{L}^{(5)}=\frac{1}{2 \sqrt{2} \pi} \frac{d-a}{d+a} \frac{\sqrt{b+d}}{d-c} .
$$

The same quantities for the second black ring are:

$$
A_{R}^{(5)}=16 \pi^{2} \sqrt{2} \frac{a^{2}(a+d)}{(a+c)} \sqrt{\frac{b+c}{(a+b)(b+d)}}, \quad T_{R}^{(5)}=\frac{1}{4 \sqrt{2} \pi} \frac{a+c}{a(a+d)} \sqrt{\frac{(a+b)(b+d)}{b+c}} .
$$


These solutions have a conical deficit for the third $\psi$-rod with $a \leq z \leq b$, with

$$
\delta=2 \pi\left(1-\sqrt{\frac{(a+b)(b+d)}{(b-a)(b+c)}}\right),
$$

which prevents the configuration from collapsing.

The ADM mass of this system, as measured at infinity is:

$$
M=\frac{3 \pi}{4}(2 a-c+d) .
$$

\section{B. 'Isotropic' coordinates and new diagrams}

\section{B.1 A coordinate transformation}

The coordinates $(\rho, z)$ defined in Section 2 have the advantage to make contact with $d=5$ Weyl coordinates and to make possible to visualise some basic properties of the solutions in terms of rod diagrams. However, although the domain of integration has a rectangular shape, the range of both $\rho$ and $z$ is unlimited and, within our numerical scheme, it is rather difficult to construct suitable meshes, especially in the $z$-direction.

In practice, we have found another coordinate system which has proven useful in the construction of some black holes with a nonspherical topology of the horizon. The transformation between $(\rho, z)$ and the new coordinates $(r, \theta)$ goes as follows. Starting with one of the diagrams in Figure 1, let us choose a finite rod there as 'central rod' (i.e. that extends from $-u \leq z \leq u$ ). Then we introduce the coordinate transformation

$$
\rho(r, \theta)=\frac{1}{2} \frac{r^{4}-r_{0}^{4}}{r^{2}} \sin 2 \theta, \quad z(r, \theta)=\frac{1}{2} \frac{r^{4}+r_{0}^{4}}{r^{2}} \cos 2 \theta,
$$

with $r_{0}^{2}=u$ and $r_{0} \leq r<\infty, 0 \leq \theta \leq \frac{\pi}{2}$, such that the 'central rod' is located at $r=r_{0}$ and all $\theta$-interval.

Then all static solutions in this work (including the $d=5$ configurations $^{26}$ ) can be studied with a metric ansatz akin to (2.8), with

$$
d s^{2}=-f_{0}(r, \theta) d t^{2}+f_{1}(r, \theta)\left(d r^{2}+r^{2} d \theta^{2}\right)+f_{2}(r, \theta) d \psi^{2}+f_{3}(r, \theta) d \Omega_{d-4}^{2} .
$$

For $r_{0}=0$ (and only two semi-infinite rods), one recovers the flat spacetime metric with $f_{0}=f_{1}=1, f_{2}=r^{2} \cos ^{2} \theta$ and $f_{3}=r^{2} \cos ^{2} \theta$. The expression of $f_{i}$ for a more complicated rod structure can easily be derived once we know the solutions in $(\rho, z)$ coordinates (note that $f_{1}$ in (B.2) does not coincide with $f_{1}$ in (2.8), since the Jacobian of the transformation (B.1) enters there also).

Also, it may be interesting to remark that $r$ and $\theta$ can be viewed as 'generalized isotropic coordinates ${ }^{27}$. This is justified by the observation that for the simplest case of

\footnotetext{
${ }^{26}$ This approach can be extended to $d=4$ axisymmetric solutions. However, in this case the transformation between the Weyl coordinates $\rho, z$ and the spherical coordinates $r, \theta$ is not given by (B.1).

${ }^{27}$ It may be interesting to notice that a version of the isotropic coordinates has been used in most of the previous numerical studies on $d=4$ asymptotically flat axisymmetric solutions, see e.g. [30].
} 
a single black hole with spherical topology of the horizon, the Schwarzschild-Tangherlini solution in isotropic coordinates is recovered (the results for $d=5$ below can easily be generalized to higher dimensions).

One of the advantages in the numerics of these coordinates is that the range of $\theta$ is finite. Moreover, the coordinate singularities in the metric functions are easier to handle in this case. For example, we have constructed in this way the full set of $d=6,7$ black objects with a $S^{2} \times S^{d-4}$ topology of the horizon, with a better accuracy than that obtained for the $(\rho, z)$ coordinate system.

The new coordinate system leads also to a new type of diagrams, which is the counterpart in $(r, \theta)$ coordinates of the rod-diagrams in Figure 1. As one can see from (B.1), $\rho=0$ corresponds to $r=r_{0}$ or $\theta=0, \pi / 2$. This suggests to show the domain of integration together with the boundary conditions satisfied by the metric functions $f_{0}, f_{2}$ and $f_{3}$. In our conventions, a wavy line indicates a horizon $f_{0}=0$, a thick line means $f_{2}=0$ (i.e. a $\psi$-rod) and a double thin line stands for an $\Omega$-rod, $f_{3}=0$, see Figure 10 (the generalization of the $d=5$ diagrams there to higher dimensions is straightforward). The horizon topology can also easily be read from that figure: a spherical horizon continues with rods of different directions, while for a black ring, the horizon continues with $\psi$-rods only.

These features are clearly illustrated by a number of $d=5$ exact solutions which we shall present in what follows.

\section{B.2 $d=5$ static solutions in 'isotropic' coordinates}

We shall start with the simplest example, corresponding to a Schwarzschild-Tangherlini black hole. The metric functions in this case read

$$
\begin{aligned}
& f_{0}(r)=\left(\frac{r^{2}-r_{0}^{2}}{r^{2}+r_{0}^{2}}\right)^{2}, \quad f_{1}(r)=\left(1+\frac{r_{0}^{2}}{r^{2}}\right)^{2}, \quad f_{2}(r, \theta)=\left(1+\frac{r_{0}^{2}}{r^{2}}\right)^{2} \cos ^{2} \theta, \\
& f_{3}(r, \theta)=\left(1+\frac{r_{0}^{2}}{r^{2}}\right)^{2} \sin ^{2} \theta
\end{aligned}
$$

with an event horizon at $r=r_{0}$, the corresponding diagram being shown in Figure 10a.

The Emparan-Reall static black ring has also a relatively simple expression in these coordinates,

$$
\begin{aligned}
& f_{0}(r)=\left(\frac{r^{2}-r_{0}^{2}}{r^{2}+r_{0}^{2}}\right)^{2}, \quad f_{2}(r, \theta)=\left(\frac{r^{2}+r_{0}^{2}}{r}\right)^{4} \frac{\sin ^{2} \theta \cos ^{2} \theta}{f_{3}(r, \theta)}, \\
& f_{3}(r, \theta)=\frac{1}{2}\left(2 R_{3}+r_{b}^{2}\left(1+\left(\frac{r_{0}}{r_{b}}\right)^{4}-\left(\frac{r_{0}}{r_{b}}\right)^{2} \cos 2 \theta\left[\left(\frac{r_{0}}{r}\right)^{2}+\left(\frac{r}{r_{0}}\right)^{2}\right]\right)\right), \\
& f_{1}(r, \theta)=\frac{r^{2}}{R_{3}}\left(\frac{1+\frac{r_{0}^{2}}{r^{2}}}{1+\frac{r_{0}^{2}}{r_{b}^{2}}}\right)^{2}\left[\left(1+\left(\frac{r_{0}}{r_{b}}\right)^{4}\right)\left(1+\left(\frac{r_{0}}{r}\right)^{4}\right)+2\left(\frac{r_{0}}{r_{b}}\right)^{2}\left(\frac{R_{3}}{r^{2}}\right)-2\left(\frac{r_{0}}{r}\right)^{2} \cos 2 \theta\right],
\end{aligned}
$$

where

$$
R_{3}=\frac{r^{2}}{2}\left[\left(1+\left(\frac{r_{b}}{r}\right)^{4}-2 \cos 2 \theta\left(\frac{r_{b}}{r}\right)^{2}\right)\left(1+\left(\frac{r_{0}^{2}}{r r_{b}}\right)^{4}-2 \cos 2 \theta\left(\frac{r_{0}^{2}}{r r_{b}}\right)^{2}\right)\right]^{1 / 2} .
$$



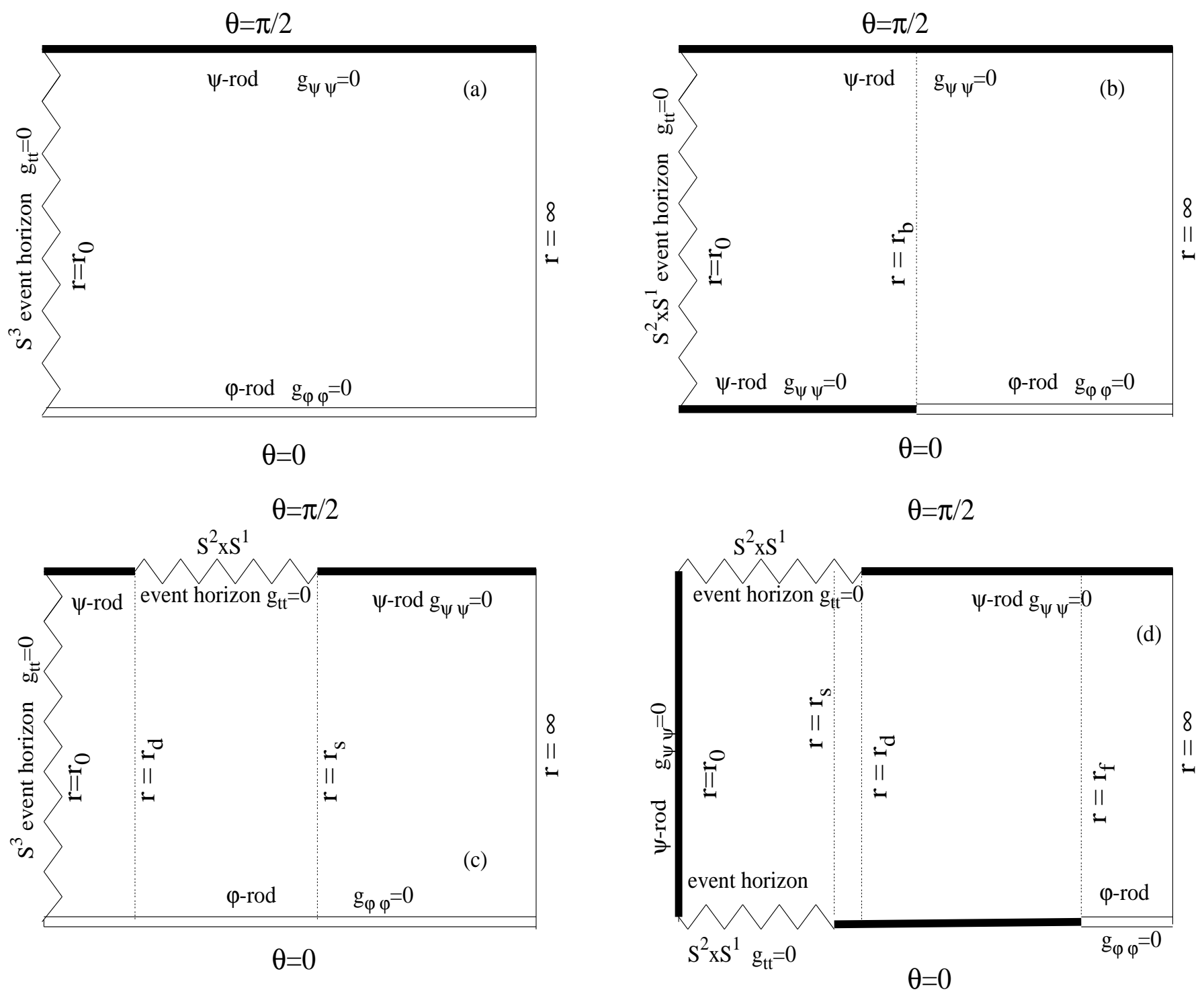

Figure 10. The domain of integration for the coordinate system (B.2) is shown for a Schwarzschild black hole, a static black ring, a black Saturn and a diring in $d=5$ dimensions.

(Note that the metric function $g_{t t}$ has no angular dependence.) The new type of roddiagram for a static black ring is shown in Figure 10b. The horizon is again located at $r=r_{0}$, the finite $\psi$-rod with an angular excess being at $\theta=0, r_{0} \leq r \leq r_{b}$.

The black Saturn can also be written in $(r, \theta)$-coordinates, with the following expression of the metric functions

$$
\begin{aligned}
& f_{0}(r, \theta)=\left(\frac{r^{2}-r_{0}^{2}}{r^{2}+r_{0}^{2}}\right)^{2} \frac{R_{1}}{R_{2}}, \quad f_{2}(r, \theta)=\frac{\left(r^{2}+r_{0}^{2}\right)^{2}}{r^{2}} \frac{R_{2}}{R_{1}} \cos ^{2} \theta, \quad f_{3}(r, \theta)=\frac{\left(r^{2}+r_{0}^{2}\right)^{2}}{r^{2}} \sin ^{2} \theta, \\
& f_{1}(r, \theta)=\left(1+\frac{r_{0}^{2}}{r^{2}}\right)^{2}\left(\frac{R_{2}+\frac{\left(r^{2}+r_{0}^{2}\right)^{2}}{r^{2}} \cos ^{2} \theta}{R_{1}+\frac{\left(r^{2}+r_{0}^{2}\right)^{2}}{r^{2}} \cos ^{2} \theta}\right)^{2} \frac{R_{1}+\frac{\left(r^{2}-r_{0}^{2}\right)^{2}}{r^{2}} \cos ^{2} \theta}{R_{2}+\frac{\left(r^{2}-r_{0}^{2}\right)^{2}}{r^{2}} \cos ^{2} \theta} \frac{\left(\rho^{2}(r, \theta)+R_{1} R_{2}\right)^{2}}{\left(\rho^{2}(r, \theta)+R_{1}^{2}\right)\left(\rho^{2}(r, \theta)+R_{2}^{2}\right)},
\end{aligned}
$$


where we have introduced the auxiliary functions

$$
\begin{aligned}
R_{1}(r, \theta)=\frac{1}{2 r^{2} r_{s}^{2}}( & -r^{2}\left(r_{0}^{4}+r_{s}^{4}\right)-r_{s}^{2}\left(r^{4}+r_{0}^{4}\right) \cos 2 \theta \\
& \left.+\sqrt{\left(r^{4}+r_{s}^{4}+2 r^{2} r_{s}^{2} \cos 2 \theta\right)\left(r_{0}^{8}+r^{4} r_{s}^{4}+2 r^{2} r_{0}^{4} r_{s}^{2} \cos 2 \theta\right)}\right), \\
R_{2}(r, \theta)=\frac{1}{2 r^{2} r_{d}^{2}}( & -r^{2}\left(r_{0}^{4}+r_{d}^{4}\right)-r_{d}^{2}\left(r^{4}+r_{0}^{4}\right) \cos 2 \theta \\
& \left.+\sqrt{\left(r^{4}+r_{d}^{4}+2 r^{2} r_{d}^{2} \cos 2 \theta\right)\left(r_{0}^{8}+r^{4} r_{d}^{4}+2 r^{2} r_{0}^{4} r_{d}^{2} \cos 2 \theta\right)}\right),
\end{aligned}
$$

and $\rho(r, \theta)$ as given by (B.1). The new rod-diagram for a static black Saturn is shown in Figure 10c. One can notice the existence of two horizons, at $r=r_{0}$ and at $\theta=\pi / 2$, $r_{d} \leq r \leq r_{s}$, respectively.

Finally, we give also the expression of the metric functions for a static di-ring in $(r, \theta)$ isotropic coordinates (the corresponding diagram is shown in Figure 10d)

$$
\begin{aligned}
& f_{0}(r, \theta)=\left(\frac{r^{2}+r_{0}^{2}}{r^{2}-r_{0}^{2}}\right)^{2} \frac{R_{1}}{R_{4}}, \quad f_{2}(r, \theta)=\frac{\left(r^{2}-r_{0}^{2}\right)^{4}}{4 r^{4}} \frac{R_{4}}{R_{1} R_{5}} \sin ^{2} 2 \theta, \quad f_{3}(r, \theta)=R_{5}, \\
& f_{1}(r, \theta)=\frac{R_{5} \sin ^{2} \theta}{r^{8}}\left(r^{2}-r_{0}^{2}\right)^{4}\left(r^{2}+r_{0}^{2}\right)^{2}\left(\frac{R_{1}+\frac{\left(r^{2}+r_{0}^{2}\right)^{2}}{r^{2}} \cos ^{2} \theta}{R_{1}+\frac{\left(r^{2}-r_{0}^{2}\right)^{2}}{r^{2}} \cos ^{2} \theta}\right)^{2}\left(\frac{R_{4}+\frac{\left(r^{2}-r_{0}^{2}\right)^{2}}{r^{2}} \cos ^{2} \theta}{R_{4}+\frac{\left(r^{2}+r_{0}^{2}\right)^{2}}{r^{2}} \cos ^{2} \theta}\right)^{2}(\mathrm{~B} .6) \\
& \quad \times\left(\frac{R_{5}+\frac{\left(r^{2}+r_{0}^{2}\right)^{2}}{r^{2}} \cos ^{2} \theta}{R_{5}^{2}+\rho^{2}(r, \theta)}\right) \frac{\left(R_{4} R_{5}+\rho^{2}(r, \theta)\right)\left(R_{1} R_{4}+\rho^{2}(r, \theta)\right)^{2}}{\left(R_{1}^{2}+\rho^{2}(r, \theta)\right)\left(R_{4}^{2}+\rho^{2}(r, \theta)\left(R_{1} R_{5}+\rho^{2}(r, \theta)\right)\left(R_{3} R_{5}+\rho^{2}(r, \theta)\right)\right.}
\end{aligned}
$$

The auxiliary functions $R_{i}$ have the following expression

$$
\begin{aligned}
R_{1}(r, \theta)=\frac{1}{2 r^{2} r_{s}^{2}}( & -r^{2}\left(r_{0}^{4}+r_{s}^{4}\right)-\left(r^{4}+r_{0}^{4}\right) r_{s}^{2} \cos 2 \theta \\
& \left.+\sqrt{\left(r^{4}+r_{s}^{4}+2 r^{2} r_{s}^{2} \cos 2 \theta\right)\left(r_{0}^{8}+r^{4} r_{s}^{4}+2 r^{2} r_{0}^{4} r_{s}^{2} \cos 2 \theta\right)}\right) \\
R_{4}(r, \theta)=\frac{1}{2 r^{2} r_{d}^{2}}\left(r^{2}\left(r_{0}^{4}+r_{d}^{4}\right)-\left(r^{4}+r_{0}^{4}\right) r_{d}^{2} \cos 2 \theta\right. & \\
& \left.+\sqrt{\left(r^{4}+r_{d}^{4}-2 r^{2} r_{d}^{2} \cos 2 \theta\right)\left(r_{0}^{8}+r^{4} r_{d}^{4}-2 r^{2} r_{d}^{4} r_{0}^{2} \cos 2 \theta\right)}\right), \\
R_{5}(r, \theta)=\frac{1}{2 r^{2} r_{f}^{2}}\left(r^{2}\left(r_{0}^{4}+r_{f}^{4}\right)-\left(r^{4}+r_{0}^{4}\right) r_{f}^{2} \cos 2 \theta\right. & \\
& \left.+\sqrt{\left(r^{4}+r_{f}^{4}-2 r^{2} r_{f}^{2} \cos 2 \theta\right)\left(r_{0}^{8}+r^{4} r_{f}^{4}-2 r^{2} r_{f}^{4} r_{0}^{2} \cos 2 \theta\right)}\right)
\end{aligned}
$$

Different from the previous cases, the line $r=r_{0}$ corresponds in this case to a finite $\psi$-rod with a conical excess. The first event horizon is located at $\theta=0, r_{0} \leq r \leq r_{s}$, and the second one at $\theta=\pi / 2, r_{0} \leq r \leq r_{d}$.

These examples make clear that the functions $f_{i}$ have a manageable expression also in $(r, \theta)$-coordinates. In particular, the singularities in the expression of $f_{1}$ at $\rho=0, z= \pm u$ 
are eliminated by the coordinate transformation (B.1). Also, it is straightforward to perform a systematic study of these solutions in these coordinates, similar to that considered in Appendix A.

\section{B.3 Rotating solutions: balanced black ring and Myers-Perry black hole}

The coordinate system introduced above turns out to be very useful also in the numerical construction of $d=5$ rotating solutions. We illustrate that by exhibiting some results for the balanced Emparan-Reall black rings and Myers-Perry black holes with a single angular momentum.

The spinning solutions can be constructed within a simple generalization of (B.2), with

$d s^{2}=-f_{0}(r, \theta) d t^{2}+\frac{1}{f_{1}(r, \theta)}\left(d r^{2}+r^{2} d \theta^{2}\right)+f_{2}(r, \theta) d \psi^{2}+f_{3}(r, \theta)\left(d \varphi+\frac{w(r, \theta)}{r} d t\right)^{2}$,

such that the horizon is located at a fixed value of $r=r_{0}$. Expanding the Einstein equations in the vicinity of the horizon in powers of $r-r_{h}$, one finds $f_{i}(r, \theta)=f_{i 0}(\theta)+f_{i 2}(\theta)\left(r-r_{h}\right)^{2}+$ $O\left(r-r_{h}\right)^{3}, w(r, \theta)=w_{h}+w_{2}(\theta)\left(r-r_{h}\right)^{2}+O\left(r-r_{h}\right)^{3}$, (where the functions $f_{i k}(\theta), w_{2}(\theta)$ are solutions of a complicated set of nonlinear second order ordinary differential equations and $f_{00}(\theta)=0$ ), which leads to an event horizon metric

$$
d \sigma^{2}=\frac{r_{h}^{2} d \theta^{2}}{f_{10}(\theta)}+f_{20}(\theta) d \psi^{2}+f_{30}(\theta) d \varphi^{2}
$$

The Hawking temperature, entropy and the event horizon velocity ${ }^{28}$ of the solutions are given by

$$
T_{H}=\frac{1}{2 \pi} \sqrt{f_{02}(\theta) f_{10}(\theta)}, \quad S=\pi^{2} r_{0} \int_{\theta=0}^{\pi / 2} d \theta \sqrt{\frac{f_{20}(\theta) f_{30}(\theta)}{f_{10}(\theta)}}, \quad \Omega_{H}=\frac{w_{h}}{r_{0}} .
$$

For any topology of the horizon, as $r \rightarrow \infty$, the Minkowski spacetime background is recovered, with $f_{0}=f_{1}=1, f_{2}=r^{2} \cos ^{2} \theta, f_{3}=r^{2} \sin ^{2} \theta, w=0$. The mass $M$ and the angular momentum $J$ of the solutions are read from the asymptotic expansion of the metric functions, $f_{0}=1-8 \pi M / 3 \pi r^{2}+\ldots, w=4 J / \pi r^{2}+\ldots$

However, the expression of the metric functions $f_{i}, w$ are quite complicated for any topology of the horizon. For example, a straightforward but cumbersome computation based on the Weyl-coordinate expressions in 18 leads to the following metric functions of a balanced black ring in the $(r, \theta)$-coordinates proposed above:

$$
\begin{aligned}
& f_{0}=\frac{1}{\left(r^{2}+r_{0}^{2}\right)^{2}} \frac{S_{1}^{2} S_{3} S_{4} S_{5}}{U_{1} Q}, \quad f_{1}=2\left(r_{b}^{4}-r_{0}^{4}\right)^{2} r^{6} \frac{R_{3}}{S_{1} S_{7}}, \quad f_{2}=-\frac{\left(r^{2}+r_{0}^{2}\right)^{2}}{2 r^{2} r_{b}^{2}\left(r^{2}-r_{0}^{2}\right)^{2}} S_{6}, \\
& f_{3}=\frac{\left(r^{2}-r_{0}^{2}\right)^{2}}{2 r^{2} r_{b}^{2}\left(r^{2}+r_{0}^{2}\right)^{2}} \frac{Q}{S_{1} S_{3}}, \quad w=-4 \sqrt{2} \frac{r^{3} r_{0}^{2} r_{b}\left(r^{2}+r_{0}^{2}\right)^{2}\left(r_{0}^{2}+r_{b}^{2}\right) \sqrt{r_{0}^{4}+r_{b}^{4}}}{\left(r^{2}-r_{0}^{2}\right)^{2}\left(r_{b}^{2}-r_{0}^{2}\right)} \frac{S_{2} S_{3}}{Q}
\end{aligned}
$$

\footnotetext{
${ }^{28}$ Note that the Killing vector $\partial / \partial t+\Omega_{H} \partial / \partial \varphi$ is null at the horizon. Also, the Einstein equation $G_{r}^{\theta}=0$ implies that the Hawking temperature is constant.
} 
where, in order to simplify the expresssions, we have defined

$$
\begin{aligned}
& S_{1}=\left(r^{4}+4 r^{2} r_{0}^{2}+r_{0}^{4}\right)\left(r_{0}^{4}+r_{b}^{4}\right)+4 r^{2} r_{0}^{4} r_{b}^{2} \cos 2 \theta-4 r^{2} r_{0}^{2} r_{b}^{2} R_{3}, \\
& S_{2}=-\left(r^{4}+r_{0}^{4}\right) r_{b}^{2}+r^{2}\left(r_{0}^{2}+r_{b}^{2}\right)^{2}-2 r^{2} r_{0}^{2} r_{b}^{2} \cos 2 \theta+2 r_{b}^{2} r^{2} R_{3}, \\
& S_{3}=\left(r^{4}-4 r^{2} r_{0}^{2}+r_{0}^{4}\right)\left(r_{0}^{4}+r_{b}^{4}\right)+4 r^{2} r_{0}^{4} r_{b}^{2} \cos 2 \theta-4 r^{2} r_{0}^{2} r_{b}^{2} R_{3}, \\
& S_{4}=\left(r_{b}^{4}+r_{0}^{4}\right) r^{2}-r_{b}^{2}\left(r^{4}+r_{0}^{4}\right) \cos 2 \theta+2 r_{b}^{2} r^{2} R_{3}, \\
& S_{5}=\left(r^{4}+4 r^{2} r_{0}^{2}+r_{0}^{4}\right)\left(r_{0}^{4}+r_{b}^{4}\right)+4 r^{2} r_{0}^{4} r_{b}^{2} \cos 2 \theta+4 r^{2} r_{0}^{2} r_{b}^{2} R_{3}, \\
& S_{6}=\left(r_{b}^{4}+r_{0}^{4}\right) r^{2}-r_{b}^{2}\left(r^{4}+r_{0}^{4}\right) \cos 2 \theta-2 r_{b}^{2} r^{2} R_{3}, \\
& S_{7}=\left(r^{4}+r_{0}^{4}\right)\left(r_{0}^{4}+r_{b}^{4}\right)-4 r^{2} r_{0}^{4} r_{b}^{2} \cos 2 \theta+4 r_{0}^{2} r_{b}^{2} r^{2} R_{3},
\end{aligned}
$$

and

$$
\begin{aligned}
& U_{1}=\left(r^{4}+r_{0}^{4}\right)\left(r_{0}^{4}-r_{b}^{4}\right)^{2}+2 r^{2} r_{0}^{2}\left(3 r_{0}^{4}+r_{b}^{4}\right)\left(r_{0}^{4}+3 r_{b}^{4}\right)+16 r^{2} r_{0}^{4} r_{b}^{2}\left(r_{0}^{4}+r_{b}^{4}\right) \cos 2 \theta, \\
& Q=S_{1}^{2} S_{4}-\frac{16 r^{2} r_{0}^{4}\left(r^{2}+r_{0}^{2}\right)^{2}\left(r_{0}^{2}+r_{b}^{2}\right)^{2}\left(r_{0}^{4}+r_{b}^{4}\right)}{\left(r^{2}-r_{0}^{2}\right)^{2}\left(r_{0}^{2}-r_{b}^{2}\right)^{2}} S_{2}^{2}, \\
& R_{3}=\frac{1}{2 r^{2} r_{b}^{2}} \sqrt{\left(r^{4}+r_{b}^{4}-2 r^{2} r_{b}^{2} \cos 2 \theta\right)\left(r_{0}^{8}+r^{4} r_{b}^{4}-2 r^{2} r_{0}^{4} r_{b}^{2} \cos 2 \theta\right)}
\end{aligned}
$$

The physical quantities are complicated functions of the input parameters $r_{0}, r_{b}$

$$
\begin{aligned}
& M=3 \pi \frac{r_{0}^{2}\left(r_{0}^{4}+r_{b}^{4}\right)}{\left(r_{0}^{2}-r_{b}^{2}\right)^{2}}, \quad J=\sqrt{2} \pi \frac{r_{0}^{2}\left(r_{0}^{2}+r_{b}^{2}\right)^{3} \sqrt{r_{0}^{4}+r_{b}^{4}}}{r_{b}\left(r_{0}^{2}-r_{b}^{2}\right)^{3}}, \quad \Omega_{H}=\frac{r_{b}\left(r_{0}^{2}-r_{b}^{2}\right)}{\sqrt{2}\left(r_{0}^{2}+r_{b}^{2}\right) \sqrt{r_{0}^{4}+r_{b}^{4}}},( \\
& T_{H}=\frac{\left(r_{0}^{2}-r_{b}^{2}\right)^{2}}{8 \sqrt{2} \pi r_{0}^{2} r_{b} \sqrt{r_{0}^{4}+r_{b}^{4}}}, \quad S=8 \sqrt{2} \pi^{2} \frac{r_{0}^{4} r_{b} \sqrt{r_{0}^{4}+r_{b}^{4}}}{\left(r_{0}^{2}-r_{b}^{2}\right)^{2}}
\end{aligned}
$$

The existence of this exact solution allows us to test the scheme developed in this work by recovering numerically the balanced black ring starting with the static solution. Then the Einstein equations are solved for the metric ansatz (B.7) and the rod structure in Figure 10b, looking for balanced solutions. Again, in practice we use a set of background functions which takes automatically into account the sets of conditions on the boundaries and determines the topology of the horizon. Therefore one defines $f_{i}=F_{i} f_{i}^{(b)}$, where $f_{i}^{(b)}$ are the functions of the static black ring as given by (B.4). In our approach, the position of the horizon $r_{0}$ and the radius of the ring $r_{b}$ are kept fixed and one varies the event horizon velocity $\Omega_{H}$. All other relevant quantities are evaluated from the numerical output. When increasing the boundary parameter $w_{h}$, the absolute value of the angular deficit excess decreases, such that $\delta$ becomes zero for a critical value of the event horizon velocity (afterwards the ring becomes over-rotating with $\delta>0$ ). By varying the value of $r_{b}$ (or the position of the horizon), the full spectrum of Emparan-Reall balanced black rings can be recovered numerically.

This approach turns out to be consistent and usually provides very good accuracy results $^{29}$. A crucial ingridient of our approach is that all numerical singularities are absorbed

\footnotetext{
${ }^{29}$ Note, however, that the black rings with a large radius or those close to the naked singularity point are difficult to obtain with enough accuracy.
} 

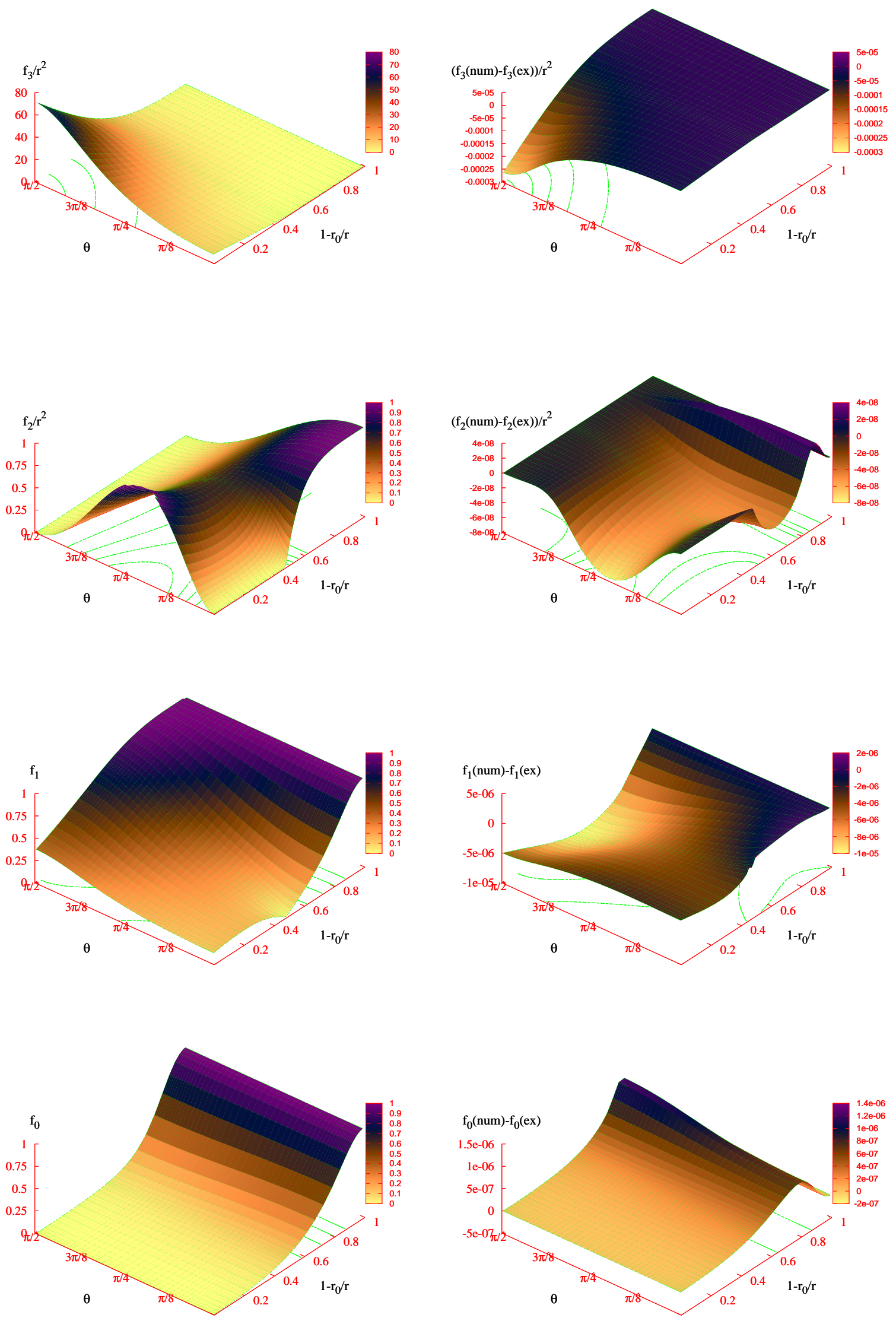

Figure 11. The metric functions $f_{i}$ as given by (B.10) together with difference between the exact solution and the numerical solution are shown for a typical $d=5$ balanced black ring. 

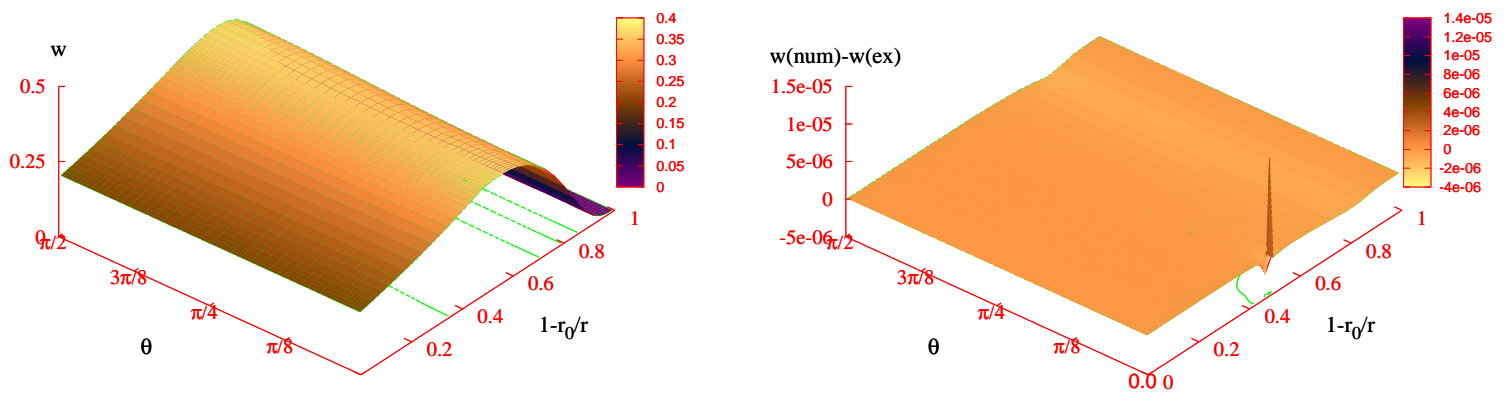

Figure 11 (continued).

already by the background functions of the static solution, such that the rotation leads to smooth functions $F_{i}, w$. The description of the numerical method presented in Section 2 is also valid in this case. In particular, we have used a compactified radial coordinate $x=1-r_{0} / r$ and a nonequidistant grid in $\theta$.

In Figure 11, we plot the metric functions of an exact solution with $r_{0}=1, r_{b}=1.93$, as well as the difference between the exact solution and the numerical result. One can see that the differences are on the order of $10^{-6}$ everywhere. As shown in Table 1, the global quantities computed numerically according to (B.9) are also in excellent agreement with the theory values (B.13).

For completeness, we give here the expression of the metric functions which enter the Myers-Perry solution with a single angular momentum within the coordinate system (B.7)

$$
\begin{aligned}
& f_{0}=\left(1-\left(\frac{r_{0}}{r}\right)^{2}\right) U, f_{1}=\left(\left(1+\left(\frac{r_{0}}{r}\right)^{2}\right)^{2}+\frac{a^{2}}{r^{2}} \cos ^{2} \theta\right)^{-1}, f_{2}=r^{2} \cos ^{2} \theta\left(1+\left(\frac{r_{0}}{r}\right)^{2}\right)^{2}, f_{3}=r^{2} \sin ^{2} \theta U, \\
& w=\frac{a}{r}\left(\frac{a^{2}}{r^{2}}+\frac{4 r_{0}^{2}}{r^{2}}\right) \frac{f_{1}}{U}, \text { and } U=\left(1+\left(\frac{r_{0}}{r}\right)^{2}\right)^{2}+\frac{a^{2}}{r^{2}}+\frac{a^{2}}{r^{2}} \frac{\left(\frac{a^{2}}{r^{2}}+\frac{4 r_{0}^{2}}{r^{2}}\right) \sin ^{2} \theta}{\left(1+\left(\frac{r_{0}}{r}\right)^{2}\right)^{2}+\frac{a^{2}}{r^{2}} \cos ^{2} \theta},
\end{aligned}
$$

the relevant quantities being given by

$$
\begin{aligned}
& M=\frac{3 \pi}{8} a\left(a^{2}+4 r_{0}^{2}\right), \quad J=\frac{\pi}{4} a\left(a^{2}+4 r_{0}^{2}\right), \quad \Omega_{H}=\frac{a}{a^{2}+4 r_{0}^{2}}, \\
& T_{H}=\frac{r_{0}}{\pi\left(a^{2}+4 r_{0}^{2}\right)}, \quad S=\pi^{2} r_{0}\left(a^{2}+4 r_{0}^{2}\right) .
\end{aligned}
$$

We have verified that within the same numerical scheme as above (with the background functions $f_{i}^{(b)}$ given by the expressions (B.3) of the Schwarzschild-Tangherlini metric in isotropic coordinates) one recovers the set of rotating black holes with an $S^{3}$ topology of the horizon and a single angular momentum. The numerical accuracy is even better in this case, since the expression of the background functions contains no square roots.

It would be interesting to recover within the same approach the balanced black Saturn and balanced black diring solutions starting with the corresponding static configurations.

To conclude, we have proposed a numerical scheme which could reproduce physically interesting $d=5$ spinning solutions starting with the corresponding static configurations. This opens the possibility to study generalizations of the Emparan-Reall balanced black 


\begin{tabular}{|r|c|c|c|c|c|c|c|}
\hline$r_{b}$ & $\Omega_{H}$ & $M($ num $)$ & $M($ ex $)$ & $\mid J($ num $) \mid$ & $\mid J($ ex $) \mid$ & $A_{H}($ num $)$ & $A_{H}($ ex $)$ \\
\hline \hline 1.61803 & 0.182574 & 24.0003 & 24.0000 & 109.547 & 109.545 & 773.616 & 773.605 \\
1.93186 & 0.204124 & 16.0000 & 16.0000 & 58.7880 & 58.7878 & 446.647 & 446.645 \\
2.18890 & 0.207020 & 13.3332 & 13.3333 & 45.0836 & 45.0843 & 332.911 & 332.909 \\
2.41421 & 0.204124 & 12.0001 & 12.0000 & 39.1922 & 39.1918 & 273.514 & 273.518 \\
2.80588 & 0.193649 & 10.6671 & 10.6667 & 34.4289 & 34.4265 & 210.552 & 210.563 \\
3.14626 & 0.182574 & 9.99982 & 10.0000 & 32.8624 & 32.8634 & 176.553 & 176.555 \\
3.45197 & 0.172516 & 9.59981 & 9.60000 & 32.4596 & 32.4607 & 154.723 & 154.726 \\
3.99215 & 0.155902 & 9.14274 & 9.14286 & 32.9869 & 32.9877 & 127.614 & 127.616 \\
4.46653 & 0.143019 & 8.88879 & 8.88889 & 34.1828 & 34.1834 & 110.970 & 110.973 \\
\hline
\end{tabular}

Table 1. The values of the event horizon velocity $\Omega_{H}$, mass parameter $M$, angular momentum $J$ and of the event horizon area $A_{H}$ are shown for rotating balanced black ring solutions with $r_{0}=1$ and several values of $r_{b}$. For comparison, both the numerical and exact values are given here.

rings and Myers-Perry black holes in various theories where closed form solutions are unlikely to exist (e.g. in Einstein-Gauss-Bonnet or Einstein-Yang-Mills theory). We hope to report on that in future work.

\section{References}

[1] R. Emparan and H. S. Reall, Phys. Rev. Lett. 88 (2002) 101101 [arXiv:hep-th/0110260].

[2] R. Emparan and H. S. Reall, Phys. Rev. D 65 (2002) 084025 [arXiv:hep-th/0110258].

[3] R. C. Myers and M. J. Perry, Annals Phys. 172 (1986) 304.

[4] H. Elvang and P. Figueras, JHEP 0705 (2007) 050 [arXiv:hep-th/0701035].

[5] H. Iguchi and T. Mishima, Phys. Rev. D 75 (2007) 064018 [arXiv:hep-th/0701043].

[6] J. Evslin and C. Krishnan, Class. Quant. Grav. 26 (2009) 125018 arXiv:0706.1231 [hep-th].

[7] H. Elvang and M. J. Rodriguez, JHEP 0804, 045 (2008) [arXiv:0712.2425 [hep-th]].

[8] M. J. Rodriguez, arXiv:1003.2411 [hep-th].

[9] R. Emparan, T. Harmark, V. Niarchos, N. A. Obers and M. J. Rodriguez, JHEP 0710 (2007) 110 [arXiv:0708.2181 [hep-th]].

[10] R. Emparan, T. Harmark, V. Niarchos and N. A. Obers, JHEP 1004, 046 (2010) [arXiv:0912.2352 [hep-th]].

[11] D. Astefanesei, M. J. Rodriguez and S. Theisen, JHEP 1008, 046 (2010) [arXiv:1003.2421 [hep-th]].

[12] B. Kleihaus, J. Kunz and E. Radu, Phys. Lett. B 678 (2009) 301 [arXiv:0904.2723 [hep-th]].

[13] G. W. Gibbons and S. W. Hawking, Phys. Rev. D 15 (1977) 2752.

[14] T. Wiseman, Class. Quant. Grav. 20 (2003) 1137 [arXiv:hep-th/0209051].

[15] G. J. Galloway and R. Schoen, Commun. Math. Phys. 266, 571 (2006) [arXiv:gr-qc/0509107]. 
[16] S. Hollands, A. Ishibashi and R. M. Wald, Commun. Math. Phys. 271, 699 (2007) [arXiv:gr-qc/0605106].

[17] T. Harmark, Phys. Rev. D 80, 024019 (2009) [arXiv:0904.4246 [hep-th]].

[18] T. Harmark, Phys. Rev. D 70 (2004) 124002 [arXiv:hep-th/0408141].

[19] S. Hollands and S. Yazadjiev, Commun. Math. Phys. 283 (2008) 749 [arXiv:0707.2775 [gr-qc]].

[20] H. Kudoh, Phys. Rev. D 75 (2007) 064006 [arXiv:gr-qc/0611136].

[21] C. Herdeiro, B. Kleihaus, J. Kunz and E. Radu, Phys. Rev. D 81 (2010) 064013 [arXiv:0912.3386 [gr-qc]];

[22] D. V. Fursaev and S. N. Solodukhin, Phys. Rev. D 52 (1995) 2133 [arXiv:hep-th/9501127].

[23] C. Herdeiro, E. Radu and C. Rebelo, Phys. Rev. D 81 (2010) 104031 [arXiv:1004.3959 [gr-qc]].

[24] T. Regge, Nuovo Cimento 19 (1961) 558.

[25] D. Astefanesei, M. J. Rodriguez and S. Theisen, JHEP 0912, 040 (2009) [arXiv:0909.0008 [hep-th]].

[26] B. Kleihaus, J. Kunz and E. Radu, JHEP 0606 (2006) 016 [arXiv:hep-th/0603119].

[27] E. Sorkin, Phys. Rev. D 74 (2006) 104027 [arXiv:gr-qc/0608115].

[28] M. Headrick, S. Kitchen and T. Wiseman, Class. Quant. Grav. 27 (2010) 035002 [arXiv:0905.1822 [gr-qc]];

H. Kudoh and T. Wiseman, Prog. Theor. Phys. 111 (2004) 475 [arXiv:hep-th/0310104];

H. Kudoh and T. Wiseman, Phys. Rev. Lett. 94 (2005) 161102 [arXiv:hep-th/0409111].

[29] W. Schönauer and R. Weiß, J. Comput. Appl. Math. 27, 279 (1989) 279;

M. Schauder, R. Weiß and W. Schönauer, The CADSOL Program Package, Universität Karlsruhe, Interner Bericht Nr. 46/92 (1992).

[30] B. Kleihaus and J. Kunz, Phys. Rev. D 57 (1998) 834 [arXiv:gr-qc/9707045];

B. Kleihaus and J. Kunz, Phys. Rev. D 57 (1998) 6138 [arXiv:gr-qc/9712086].

[31] R. Bach and H. Weyl, Math. Z 13 (1922) 134.

[32] W. Israel and K. A. Khan, Nuovo Cimento 33 (1964) 331.

[33] H. S. Tan and E. Teo, Phys. Rev. D 68 (2003) 044021 [arXiv:hep-th/0306044].

[34] H. K. Kunduri and J. Lucietti, arXiv:1002.4656 [hep-th].

[35] A. A. Pomeransky and R. A. Sen'kov, arXiv:hep-th/0612005.

[36] P. Figueras, JHEP 0507 (2005) 039 [arXiv:hep-th/0505244];

T. Mishima and H. Iguchi, Phys. Rev. D 73 (2006) 044030 [arXiv:hep-th/0504018].

[37] J. Kunz, F. Navarro-Lerida and J. Viebahn, Phys. Lett. B 639 (2006) 362 [arXiv:hep-th/0605075].

[38] H. K. Kunduri and J. Lucietti, Phys. Lett. B 609 (2005) 143 [arXiv:hep-th/0412153];

S. S. Yazadjiev, arXiv:hep-th/0507097.

[39] B. Kleihaus, J. Kunz and E. Radu, JHEP 1002 (2010) 092 [arXiv:0912.1725 [gr-qc]].

[40] F. Schwartz, arXiv:0704.2403 [gr-qc]. 